\title{
Traveling Wave Solutions of a Diffusive SEIR Epidemic Model with Nonlinear Incidence Rate
}

\author{
Lin Zhao*, Liang Zhang and Haifeng Huo
}

\begin{abstract}
This paper is concerned with the existence and nonexistence of traveling wave solutions of a diffusive SEIR epidemic model with nonlinear incidence rate, which are determined by the basic reproduction number $R_{0}$ and the minimal wave speed $c^{*}$. Namely, the system admits a nontrivial traveling wave solution if $R_{0}>1$ and $c \geq c^{*}$ and then the non-existence of traveling wave solutions of the system is established if $R_{0}>1$ and $0<c<c^{*}$. Especially, using numerical simulation, we give the basic framework of traveling wave solutions of the system.
\end{abstract}

\section{Introduction}

A tremendous number of models have been formulated, analyzed and applied to a variety of infectious diseases qualitatively and quantitatively. Mathematical models have become important tools in analyzing the spread and control of infectious diseases [1]. Ross first proposed a system of ordinary differential equations which is the origin of the most epidemiological models especially for the modern susceptible-infected-recovered (SIR) compartmental model. In fact, some infectious diseases have a latency: the infected individuals do not infect other susceptible individuals immediately and remain in the exposed class before becoming infective, such as AIDS/HIV, human tuberculosis or bovine tuberculosis etc, it may take months for the infection to develop to the infectious stage [4]. In order for a model to be more realistic, it is essential for introduction of the exposed group (latent group) in a epidemic model, for example an SEIR or SEIRS epidemic model etc. Up to now, there have been many studies on the epidemic model with the exposed group, see $2,5,12,22,23$ and the references therein.

Functional form of the incidence rate can have a crucial role for modeling of epidemic dynamics 15]. In classical epidemiological models, the bilinear incidence rate is used,

Received May 5, 2018; Accepted October 24, 2018.

Communicated by Eiji Yanagida.

2010 Mathematics Subject Classification. 35C07, 35B40, 35K57, 92D30.

Key words and phrases. SEIR epidemic model, nonlinear incidence rate, the basic reproduction number, the minimal speed, traveling wave solutions.

Zhao was supported by National Natural Science Foundation of China (11801244), the HongLiu first class disciplines development program of Lanzhou University of Technology.

*Corresponding author. 
namely, proportional to the product of the number of infective individuals which is denoted by $I$ and the number of susceptible individuals which is expressed by $S$, which indicates that the number of infected individuals can grow indefinitely, while it is not the case. In addition, a fraction $S / S+I$ of these contacts is with susceptible individuals, and hence the total number of new infections is $I S / S+I$. However, there is a variety of reasons why this standard bilinear incidence rate may require modification, e.g., the underlying assumption of homogeneous mixing may be invalid [19]. To solve it, Korobeinikov and Maini [19] used an arbitrary function $f(S, I, S+I)$ instead of other incidence rates and thought that due to arbitrariness of the incidence rate, such system can describe various of infectious diseases and it seems to be reasonable to consider some properties of the system, where $f(S, I, S+I)$ satisfies $f(S, 0, N)=f(0, I, N)=0(N:=S+I), \partial f(S, I, N) / \partial S>0, \partial f(S, I, N) / \partial I>0$ and $\partial^{2} f(S, I, N) / \partial I^{2}<0$ for all $S, I \geq 0$. Then they analyzed the impact of the form of the non-linearity of the infectious disease incidence rate on the dynamics of epidemiological models, that is, a sufficient condition for stability of the endemic equilibrium state is the concavity of the nonlinear incidence rate with respect to the number of infective individuals. For further developments, we refer to Korobeinikov [16, 17] and the cited references therein. Huang et al. 15] established global stability for delay SIR and SEIR epidemic models which are incorporated into the constant recruitment and the nonlinear incidence rate defining the general form $F(S) G(I)$. In addition, $F(s)$ and $G(i)$ are always positive, continuous, and monotonically increasing for all $s>0$ and $i>0, F(0)=G(0)=0$ and $\partial^{2} G(I) / \partial I^{2}<0$ for all $S, I \geq 0$. Dubey [7] took into account dynamics of an SEIR epidemic model combing with the constant recruitment and the incidence rate on Holling type II, III and IV. In addition, there have been other papers studying epidemic models with the nonlinear incidence rate, see $[21,25,26,35,38]$ and the references therein.

A special class of analytical solutions named traveling wave solutions for the spatial transmission of infectious diseases has a lot of importance, because various infectious diseases can be well described by the mathematical model with spatial effects that can give rise to a moving zone of transition from an infective state to a diseases-free state in general. Up to now, many studies focus on establishing the existence of traveling wave solutions and the asymptotic speed of propagation in epidemic models, see $3,6,8,9,13$, $14,20,23,24,31,32,34,39,40$ and the references therein. For a diffusive SEIR model, Tian and Yuan 28] established the existence and nonexistence of traveling waves of the system with nonlocal reaction, precisely, there exists a positive constant number $c^{*}$ such that if the basic reproduction number $R_{0}$ is larger than one, then the system can admit a nontrivial and non-negative traveling wave solution satisfying the corresponding boundary conditions for any wave speed $c>c^{*}$ by the the Schauder fixed point theorem and the limit argument, and if $R_{0} \leq 1$ or $R_{0}>1$ and $0<c<c^{*}$, there exists no nontrivial 
and nonnegative traveling wave solution on the system by using Laplace transform. For further developments, we refer to Tian and Yuan [27, 29]. Xu [36] proposed a diffusive SEIR epidemic model with saturating incidence rate which has the form of $S g(I)$ and $g$ is a continuous function with $I$ and analyzed the existence and nonexistence of traveling wave solutions of the system by using the Schauder fixed point theorem and the Laplace transform. In addition, Xu [37] proposed a simple diffusive SEIR epidemic model where the total population is variable and considered the existence and nonexistence of traveling wave solutions connecting two equilibria which is determined by the basic reproduction number. Wang and $\mathrm{Xu}$ [33] investigated and simulated the existence of traveling wave solutions of a diffusion SEIR epidemic model with relapse, where they used the bilinear incidence rate. Namely, by applying a pair of upper-lower solutions, the cross-iteration method and the Schauder's fixed point theorem, they obtained the existence of travelling wave solutions of the system.

The purpose of this paper is to incorporate spatial heterogeneity, the nonlinear incidence rate, the constant recruitment and the rate of the amelioration into the SEIR disease model and to investigate the existence and non-existence of the traveling wave solutions of the epidemic model. There have been some studies on the traveling wave solution of a diffusive SEIR epidemic model. However, there are few results about their combined effects on an SEIR epidemic model. Precisely, we consider the following SEIR epidemic model:

$$
\begin{aligned}
& \frac{\partial u(t, x)}{\partial t}=d \Delta u(t, x)+\mu-\beta g(u(t, x)) w(t, x)-\lambda u(t, x), \\
& \frac{\partial v(t, x)}{\partial t}=D_{v} \Delta v(t, x)+[\beta g(u(t, x))+r] w(t, x)-\sigma v(t, x), \quad t>0, x \in \mathbb{R}, \\
& \frac{\partial w(t, x)}{\partial t}=D \Delta w(t, x)+\gamma v(t, x)-\delta w(t, x),
\end{aligned}
$$

in addition, the equation for the recovered group,

$$
\frac{\partial r(t, x)}{\partial t}=\mathcal{T} \Delta r(t, x)-\kappa r(t, x)+\widetilde{m} w(t, x),
$$

is omitted, where $u, v, w$ and $r$ represent the susceptible compartment, the latent compartment, infectious compartment and the recovered compartment, respectively. The susceptible class includes the individuals that can be infected by the infectious disease; the individuals who have been infected and not have an influence on other susceptible individuals constitute the latent group; the infective individuals consist of those who have capable of infecting others and the individuals with full immunity, or isolated, or sadly dead is included by the removed group. $\mu, \lambda, \beta, r, \sigma, \gamma, \kappa, \widetilde{m}, d, D_{v}, D$ and $\mathcal{T}$ are positive constants and $d \geq D_{v} \geq D$, and $d, D_{v}, D$ and $\mathcal{T}$ are the diffusive rates of the susceptible individuals, the latent individuals, the infectious individuals and the recovered 
individuals, respectively. In (1.1), $\sigma(\sigma>\gamma)$ and $\kappa$ represent the rate that the exposed hosts leave $v$-compartment and $r$-compartment, respectively, $\delta$ is the sum of the mortality rate, the rate of the amelioration $r(r<\delta), \mu$ denotes the entering flux of susceptible individuals, $\widetilde{m}(\widetilde{m}<\delta)$ is the recovered rate, $\lambda$ denotes the rate which individuals leave or die the population, and $\beta$ is the infection contamination rate. Here transmission rate of the infection is assumed by the nonlinear incidence rate $\beta g(u) w$ and $g(u)$ satisfies the following assumptions:

(i) $g(u): \mathbb{R}^{+} \rightarrow \mathbb{R}^{+}$can be continuously differentiable for all $u \geq 0$;

(ii) $g(0)=0, g^{\prime}(0) \geq 0$ and $g^{\prime}(u)>0$ for all $u \in(0, \infty)$;

(iii) There exists $\eta>0$ such that $g(u) \leq \eta u$ for all $u \geq 0$.

Note that the class of $g(u)$ satisfying (H1) include many common incidence functions such as $g(u)=u, g(u)=u /(b u+a), g(u)=u^{2} /\left(c+d u^{2}\right)$ etc., which $a, b, c$ and $d$ are positive constants (see 7,10 ).

The main results of this paper contain the existence and nonexistence of traveling wave solutions of 1.1 connecting two equilibria. The plan of this work is as follows. In Section 2, when $R_{0}>1$, we show that there exists a $c^{*}>0$ such that for any $c \geq c^{*}$, system (1.1) admits a nontrivial traveling wave solution connecting two equilibria with wave speed $c$. In Section 3, making use of the two-sided Laplace transform, we show that system (1.1) admits no nontrivial traveling waves for $R_{0}>1$ and $0<c<c^{*}$. In addition, we simulate the existence of traveling wave solutions of system (1.1), which give the basic framework of the traveling wave solutions of system (1.1).

\section{Main results}

To discuss traveling wave solutions of 1.1 , we need to look for constant equilibria and the basic reproduction number of (1.1). It is equivalent to find those on the corresponding ODE system

$$
\frac{d u}{d t}=\mu-\beta g(u) w-\lambda u, \quad \frac{d v}{d t}=[\beta g(u)+r] w-\sigma v, \quad \frac{\partial w}{\partial t}=\gamma v-\delta w .
$$

Obviously, $\left(u^{0}, v^{0}, w^{0}\right)\left(u^{0}=\mu / \lambda\right.$ and $\left.v^{0}=w^{0}=0\right)$ is a disease-free equilibrium of 2.1. From [30], the basic reproduction number of system (2.1) at the disease free equilibrium $\left(u^{0}, v^{0}, w^{0}\right)$ can be defined by

$$
R_{0}:=\frac{\gamma(\beta g(\mu / \lambda)+r)}{\sigma \delta}
$$

Since $\frac{\gamma(\beta g(\mu / \lambda)+r)}{\sigma \delta}>1, \gamma r /(\sigma \delta)<1(\gamma<\sigma, r<\delta)$ and (ii) of (H1), there exists $u^{*} \in$ $(0, \mu / \lambda)$ such that $g\left(u^{*}\right):=\frac{\sigma-r \gamma / \delta}{\beta \gamma / \delta}$. Using a straightforward computation, system 2.1) 
admits a positive unique endemic equilibrium $\left(u^{*}, v^{*}, w^{*}\right)$ which results in

$$
g\left(u^{*}\right):=\frac{\sigma-r \gamma / \delta}{\beta \gamma / \delta}, \quad v^{*}:=\frac{\mu-\lambda u^{*}}{\sigma-r \gamma / \delta}, \quad w^{*}:=\frac{\gamma v^{*}}{\delta} .
$$

By the similar arguments as those in [18, it is shown that when $R_{0} \leq 1$ the disease-free equilibrium $\left(u_{0}, v_{0}, w_{0}\right)=(\mu / \lambda, 0,0)$ is globally asymptotically stable, and when $R_{0}>$ 1 , the unique endemic disease equilibrium $\left(u^{*}, v^{*}, w^{*}\right)$ is globally asymptotically stable. Consequently, system (1.1) also admits two equilibria, that is, the disease free equilibrium $\left(u^{0}, v^{0}, w^{0}\right)$ and the endemic equilibrium $\left(u^{*}, v^{*}, w^{*}\right)$.

A traveling wave solution of (1.1) takes the special form as bellows

$$
(u(\xi), v(\xi), w(\xi)), \quad \xi=x+c t \in \mathbb{R}
$$

where the parameter $c$ is called the wave speed and $\xi=x+c t$ is the moving coordinate. For a convenience of calculation, let $\lambda=\mu$ in system (1.1). Plugging (2.3) into (1.1), we can get the wave form equations:

$$
\begin{aligned}
& d u^{\prime \prime}(\xi)-c u^{\prime}(\xi)+\mu-\beta g(u(\xi)) w(\xi)-\mu u(\xi)=0, \\
& D_{v} v^{\prime \prime}(\xi)-c v^{\prime}(\xi)+\beta g(u(\xi)) w(\xi)+r v(\xi)-\sigma v(\xi)=0, \quad \xi \in \mathbb{R} . \\
& D w^{\prime \prime}(\xi)-c w^{\prime}(\xi)+\gamma v(\xi)-\delta w(\xi)=0
\end{aligned}
$$

The main aim of the paper is to look for a nonnegative and nontrivial solution $(u(\xi), v(\xi)$, $w(\xi))$ of 2.4 , which is supplemented with the boundary conditions as bellows

$$
\begin{aligned}
& u(-\infty)=1, \quad u(+\infty)=u^{*}, \quad v(-\infty)=0, \\
& v(+\infty)=v^{*}, \quad w(-\infty)=0, \quad w(+\infty)=w^{*},
\end{aligned}
$$

where $u^{*}, v^{*}$ and $w^{*}$ are defined in 2.2.

From 2.3), it is easy to see that there is a close relationship between existence of a traveling wave solution of (1.1) and the wave speed $c$. Thus, we analyze the properties of the wave speed $c$ here. First, we need to a linearized system which can be achieved through linearizing the $v$-th equation and $w$-th equation of $(2.4)$ at $(1,0,0)$

$$
\begin{aligned}
& D_{v} v^{\prime \prime}(\xi)-c v^{\prime}(\xi)+\beta g(1) w(\xi)+r w(\xi)-\sigma v(\xi)=0 \\
& D w^{\prime \prime}(\xi)-c w^{\prime}(\xi)+\gamma v(\xi)-\delta w(\xi)=0
\end{aligned}
$$

Plugging the trial function $(v, w)(\xi)=(p, q) e^{\lambda \xi}$ into 2.6$)$, we get the characteristic equations

$$
D_{v} p \lambda^{2}-c p \lambda-\sigma p+(\beta g(1)+r) q=0, \quad D q \lambda^{2}-c q \lambda-\delta q+\gamma p=0
$$


Set

$$
\mathcal{D}=\left[\begin{array}{cc}
D_{v} & 0 \\
0 & D
\end{array}\right], \quad \mathcal{C}=c \mathcal{I}, \quad \mathcal{F}=\left[\begin{array}{cc}
0 & \beta g(1)+r \\
\gamma & 0
\end{array}\right], \quad \mathcal{V}=\left[\begin{array}{ll}
\sigma & 0 \\
0 & \delta
\end{array}\right]
$$

where $\mathcal{I}$ is the identity matrix. Let $\Lambda(\lambda, c)=\mathcal{D} \lambda^{2}-\mathcal{C} \lambda+\mathcal{F}-\mathcal{V}$ and $\mathcal{Q}=(p, q)^{T}$, then (2.7) can simplify

$$
\Lambda(\lambda, c) \mathcal{Q}=0
$$

Define $\check{D}=\mathcal{V}^{-1} \mathcal{D}, \check{C}=\mathcal{V}^{-1} \mathcal{C}$ and $\check{F}=\mathcal{V}^{-1} \mathcal{F}, 2.8$ can reduce to

$$
\mathcal{H}(\lambda, c) \mathcal{Q}=\mathcal{Q}
$$

where $\mathcal{H}(\lambda, c):=\left(-A \lambda^{2}+B \lambda+\mathcal{I}\right)^{-1} F$ can be derived by

$$
\mathcal{H}(\lambda, c)=\left[\begin{array}{cc}
0 & \frac{\beta g(1)+r}{m_{0}(\lambda, c)} \\
\frac{\gamma}{m_{1}(\lambda, c)} & 0
\end{array}\right]
$$

where $m_{0}(\lambda, c)=-D_{v} \lambda^{2}+c \lambda+\sigma$ and $m_{1}(\lambda, c)=-D \lambda^{2}+c \lambda+\delta$.

Let $\rho(\lambda, c)$ be the principle eigenvalue of $\mathcal{H}(\lambda, c)$. Using a straightforward computation, we get the following expression

$$
\rho(\lambda, c)=\left[\frac{(\beta g(1)+r) \gamma}{m_{0}(\lambda, c) m_{1}(\lambda, c)}\right]^{1 / 2}, \quad \forall \lambda \in\left(0, \lambda_{\max }(c)\right), c \geq 0,
$$

where $\lambda_{\max }(c)=\min \left\{\left(c+\sqrt{c^{2}+4 D_{v} \sigma}\right) /\left(2 D_{v}\right),\left(c+\sqrt{c^{2}+4 D \delta}\right) /(2 D)\right\}$.

Secondly, similar to 39 , Section 2], we can obtain the following lemmas.

Lemma 2.1. Let

$$
R_{0}=\frac{\gamma}{\sigma \delta}(\beta g(1)+r)>1
$$

Then there exist $c^{*}>0$ and $\lambda^{*} \in\left(0, \lambda_{\max }(c)\right)$ satisfying

(i) $\rho(\lambda, c)>1, \forall 0 \leq c<c^{*}$ and $\forall \lambda \in\left(0, \lambda_{\max }(c)\right)$;

(ii) $\rho\left(\lambda^{*}, c^{*}\right)=1, \rho\left(\lambda, c^{*}\right)>1$ for $\lambda \in\left(0, \lambda^{*}\right)$ and $\rho\left(\lambda, c^{*}\right)>1$ for $\lambda \in\left(\lambda^{*}, \lambda_{\max }\left(c^{*}\right)\right)$;

(iii) if $c>c^{*}$, then $\rho(\lambda, c)=1$ has exactly two positive real roots $\lambda_{1}(c)$ and $\lambda_{2}(c)$ with $0<\lambda_{1}(c)<\lambda_{2}(c)<\lambda_{\max }(c)$ such that $\frac{\partial}{\partial \lambda} \rho\left(\lambda_{1}(c), c\right)<0$, $\frac{\partial}{\partial \lambda} \rho\left(\lambda_{2}(c), c\right)>0$. In particular, there exists a unit vector $\mathcal{Q}(c)=(p(c), q(c))^{T} \in \mathbb{R}^{2}$ with positive components such that

$$
\operatorname{det} \Lambda\left(\lambda_{1}(c), c\right)=0 \quad \text { and } \quad \Lambda\left(\lambda_{1}(c), c\right) \mathcal{Q}(c)=0 .
$$


Remark 2.2. For $c>c^{*}$, let $\nu>0$ be small enough with $\lambda_{1}(c)+\nu \in\left(\lambda_{1}(c), \lambda_{2}(c)\right)$. In view of Lemma 2.1, there exists the positive vector $\zeta(c)=\left(\zeta_{1}(c), \zeta_{2}(c)\right)^{T}$ satisfying

$$
\mathcal{H}\left(\lambda_{1}(c)+\nu, c\right) \zeta(c)=\rho\left(\lambda_{1}(c)+\nu, c\right) \zeta(c)<\zeta(c)
$$

which implies that

$$
\Lambda\left(\lambda_{1}(c)+\nu, c\right) \zeta(c)<0 .
$$

Now, the main conclusion we shall obtain reads as follows.

Theorem 2.3. Let (H1) be satisfied. The following results hold true:

(i) If $R_{0}>1$ and $c \geq c^{*}$, then system (2.4) and 2.5 admits a solution $(u(\cdot), v(\cdot), w(\cdot))$.

(ii) If $R_{0}>1$ and $0<c<c^{*}$, then there exists no nontrivial solution $(u(x+c t), v(x+$ $c t), w(x+c t))$ of system (2.4) satisfying (2.5).

In general, $c^{*}$ is called by the minimal wave speed.

$$
\text { 3. Proof of assertion (i) in Theorem } 2.3
$$

In the section, we show assertion (i) of Theorem 2.3. For this purpose, we divide into the following two subsections.

\subsection{Case I: $R_{0}>1$ and $c>c^{*}$}

First, we need to prove that system (2.4) and (2.5) admits a solution $(u(\cdot), v(\cdot), w(\cdot))$ if $R_{0}>1$ and $c>c^{*}$. In this subsection, assume $R_{0}>1$ and $c>c^{*}$. In addition, for the sake of simplicity,

$$
\lambda_{1}(c), \quad \mathcal{Q}(c)=(p(c), q(c))^{T} \quad \text { and } \quad \zeta(c)=\left(\zeta_{1}(c), \zeta_{2}(c)\right)^{T},
$$

which is defined in Lemma 2.1 and Remark 2.2 are substituted by $\lambda_{c}, \mathcal{Q}=(p, q)^{T}$ and $\zeta=\left(\zeta_{1}, \zeta_{2}\right)^{T}$. It further follows that

$$
\Lambda\left(\lambda_{c}, c\right) \mathcal{Q}=0, \quad \Lambda\left(\lambda_{c}+\nu, c\right) \zeta<0
$$

At first, by using Lemma 2.1 and Remark 2.2, we construct the super- and sub-solutions of system (2.4).

Lemma 3.1. The vector valued map $M(\xi)=\left(\widetilde{M}_{1}(\xi), \widetilde{M}_{2}(\xi)\right)$ with $\widetilde{M}_{1}(\xi)=$ pe $e_{c} \xi$ and $\widetilde{M}_{2}(\xi)=q e^{\lambda_{c} \xi}$ satisfies the following equations

$$
\begin{gathered}
D_{v} \widetilde{M}_{1}^{\prime \prime}(\xi)-c \widetilde{M}_{1}^{\prime}(\xi)+(\beta g(1)+r) \widetilde{M}_{2}(\xi)-\sigma \widetilde{M}_{1}(\xi)=0 \\
D \widetilde{M}_{2}^{\prime \prime}(\xi)-c \widetilde{M}_{2}^{\prime}(\xi)+\gamma \widetilde{M}_{2}(\xi)-\delta \widetilde{M}_{1}(\xi)=0
\end{gathered}
$$


Lemma 3.2. For every $\theta>0$ small enough with $\theta<\min \left\{\lambda_{c}, c / d\right\}$ and $K>1$ sufficiently large, the map $\widetilde{F}(\xi)=\max \left\{1-K e^{\theta \xi}, 0\right\}$ satisfies

$$
c \widetilde{F}^{\prime}(\xi) \leq d \widetilde{F}^{\prime \prime}(\xi)+\mu-\mu \widetilde{F}(\xi)-\beta g(\widetilde{F}(\xi)) \widetilde{M}_{2}(\xi)
$$

with $\xi \in \mathbb{R}$ and $\xi \neq-\frac{1}{\theta} \ln K$.

Proof. To get it, we analyze the following two cases.

Case 1. If $\xi>-\frac{1}{\theta} \ln K$, the inequality 3.3 holds.

Case 2. If $\xi<-\frac{1}{\theta} \ln K$, then one gets $\widetilde{M}_{1}(\xi)=p e^{\lambda_{c} \xi}, \widetilde{M}_{2}(\xi)=q e^{\lambda_{c} \xi}$ and $\widetilde{F}(\xi)=$ $1-K e^{\theta \xi}$. Furthermore, it follows from $\theta<c / d$ and $e^{-\frac{\lambda_{c}-\theta}{\theta} \ln K} \rightarrow 0$ as $K \rightarrow+\infty$ that

$$
\begin{aligned}
& -d K \theta^{2} e^{\theta \xi}+c K \theta e^{\theta \xi}-\beta g\left(\left(1-K e^{\theta \xi}\right)\right) q e^{\lambda_{c} \xi}-\mu\left(1-K e^{\theta \xi}\right)+\mu \\
= & -d K \theta^{2} e^{\theta \xi}+c K \theta e^{\theta \xi}-\beta g\left(\left(1-K e^{\theta \xi}\right)\right) q e^{\lambda_{c} \xi}+K \mu e^{\theta \xi} \\
= & {\left[(c-d \theta) K \theta-\beta g\left(\left(1-K e^{\theta \xi}\right)\right) q e^{\left(\lambda_{c}-\theta\right) \xi}+K \mu\right] e^{\theta \xi} } \\
\geq & {\left[(c-d \theta) K \theta-\beta g(1) q e^{-\frac{\lambda_{c}-\theta}{\theta} \ln K}+K \mu\right] e^{\theta \xi} } \\
\geq & 0 .
\end{aligned}
$$

Thus, the inequality 3.3 holds for $K>1$ large enough. This completes the proof.

Lemma 3.3. Fix $0<\omega<\theta$. Then the function $\widetilde{H}(\xi)=\left(h_{1}(\xi), h_{2}(\xi)\right)$ with $h_{1}(\xi)=$ $\max \left\{p e^{\lambda_{c} \xi}-B \zeta_{1} e^{\left(\lambda_{c}+\omega\right) \xi}, 0\right\}$ and $h_{2}(\xi)=\max \left\{q e^{\lambda_{c} \xi}-B \zeta_{2} e^{\left(\lambda_{c}+\omega\right) \xi}, 0\right\}$ satisfies

$$
c h_{1}^{\prime}(\xi) \leq D_{v} h_{1}^{\prime \prime}(\xi)+\beta h_{2}(\xi) g(\widetilde{F}(\xi))+r h_{2}(\xi)-\sigma h_{1}(\xi), \quad \forall \xi<\frac{1}{\omega} \ln \frac{p}{B \zeta_{1}}
$$

and

$$
c h_{2}^{\prime}(\xi) \leq D h_{2}^{\prime \prime}(\xi)+\gamma h_{1}(\xi)-\delta h_{2}(\xi), \quad \forall \xi<\frac{1}{\omega} \ln \frac{q}{B \zeta_{2}}
$$

where $B>1$ is sufficiently large such that $\max \left\{\frac{1}{\omega} \ln \frac{p}{B \zeta_{1}}, \frac{1}{\omega} \ln \frac{q}{B \zeta_{2}}\right\}<\frac{1}{\theta} \ln \frac{1}{K}$.

Proof. When $\xi<\frac{1}{\omega} \ln \frac{p}{B \zeta_{1}}$, then one has $h_{1}(\xi)=p e^{\lambda_{c} \xi}-B \zeta_{1} e^{\left(\lambda_{c}+\omega\right) \xi}$ and $\widetilde{F}(\xi)=1-K e^{\theta \xi}$. Plugging $h_{1}(\xi)$ into inequality 3.4 for $\xi<\frac{1}{\omega} \ln \frac{p}{B \zeta_{1}}$, we get the following inequality

$$
\begin{aligned}
& B\left[-D_{v} \zeta_{1}\left(\lambda_{c}+\omega\right)^{2}+c \zeta_{1}\left(\lambda_{c}+\omega\right)-r \zeta_{1}+\sigma \zeta_{2}\right] e^{\left(\lambda_{c}+\omega\right) \xi}+\beta g(\widetilde{F}(\xi)) h_{2}(\xi) \\
& +D_{v} p \lambda_{c}^{2} e^{\lambda_{c} \xi}-c p \lambda_{c} e^{\lambda_{c} \xi}+r p e^{\lambda_{c} \xi}-\sigma q e^{\lambda_{c} \xi} \\
= & B\left[-D_{v} \zeta_{1}\left(\lambda_{c}+\omega\right)^{2}+c \zeta_{1}\left(\lambda_{c}+\omega\right)-r \zeta_{1}+\sigma \zeta_{2}\right] e^{\left(\lambda_{c}+\omega\right) \xi}+\beta g(\widetilde{F}(\xi)) h_{2}(\xi)-\beta g(1) q e^{\lambda_{c} \xi} \\
\geq & 0 .
\end{aligned}
$$

In view of (H1) and Taylor's theorem, we deduce that there exists a positive constant $K_{1}$ satisfying

$$
g(\widetilde{F}(\xi))=g(1)-g^{\prime}(1) K e^{\theta \xi}+R_{1}(\xi)
$$


with

$$
\left|R_{1}(\xi)\right| \leq K_{1} e^{2 \theta \xi}
$$

It follows that

$$
\begin{aligned}
& \beta g(\widetilde{F}(\xi)) h_{2}(\xi)-\beta g(1) q e^{\lambda_{c} \xi} \\
= & \beta\left(g(1)-g^{\prime}(1) K e^{\theta \xi}+R_{1}(\xi)\right)\left(q e^{\lambda_{c} \xi}-B \zeta_{2} e^{\left(\lambda_{c}+\omega\right) \xi}\right)-\beta g(1) q e^{\lambda_{c} \xi} \\
= & -B \beta g(1) \zeta_{2} e^{\left(\lambda_{c}+\omega\right) \xi}-\beta g^{\prime}(1) K e^{\theta \xi} h_{2}(\xi)+\beta h_{2}(\xi) R_{1}(\xi) .
\end{aligned}
$$

Therefore, we only need to prove

$$
\begin{aligned}
& B\left[-D_{v} \zeta_{1}\left(\lambda_{c}+\omega\right)^{2}+c \zeta_{1}\left(\lambda_{c}+\omega\right)-\beta g(1) \zeta_{2}-r \zeta_{1}+\sigma \zeta_{2}\right] e^{\left(\lambda_{c}+\omega\right) \xi} \\
& -\beta g^{\prime}(1) k e^{\theta \xi} h_{2}(\xi)+\beta R_{1}(\xi) h_{2}(\xi) \\
\geq & \left\{B\left[-D_{v} \zeta_{1}\left(\lambda_{c}+\omega\right)^{2}+c \zeta_{1}\left(\lambda_{c}+\omega\right)-\beta g(1) \zeta_{2}-r \zeta_{1}+\sigma \zeta_{2}\right]\right. \\
& \left.\quad-\beta g^{\prime}(1) K q e^{(\theta-\omega) \xi}-\beta K_{1} q e^{(2 \theta-\omega) \xi}\right\} e^{\left(\lambda_{c}+\omega\right) \xi}
\end{aligned}
$$$$
\geq 0 \text {. }
$$

Following from $0<\omega<\theta$, it has

$$
e^{(\theta-\omega) \xi}<{\frac{p}{B \zeta_{1}}}^{\frac{(\theta-\omega)}{\omega}} \rightarrow 0 \text { and } \quad e^{(2 \theta-\omega) \xi}<{\frac{p}{B \zeta_{1}}}^{\frac{(2 \theta-\omega)}{\omega}} \rightarrow 0 \quad \text { as } B \rightarrow+\infty,
$$

which can imply that the inequality 3.6 holds for $B>1$ large enough. The inequality 3.5 can be treated similarly. The proof is completed.

Secondly, let $\mathcal{J}:=\max \left\{\frac{1}{\omega} \ln \frac{B \zeta_{1}}{p}, \frac{1}{\omega} \ln \frac{B \zeta_{2}}{q}\right\}$ and $X>\mathcal{J}$. Let

$$
\Gamma_{X}=\left\{\begin{array}{c}
\left(\chi(\cdot), \varphi_{1}(\cdot), \varphi_{2}(\cdot)\right) \in C\left([-X, X], \mathbb{R}^{3}\right): \chi( \pm X)=\widetilde{F}( \pm X), \widetilde{F}(\xi) \leq \chi(\xi) \leq 1, \\
\varphi_{i}( \pm X)=h_{i}( \pm X), h_{i}(\xi) \leq \varphi_{i}(\xi) \leq \widetilde{M}_{i}(\xi), i=1,2
\end{array}\right\}
$$

Next, we consider the boundary valued problem as follows

$$
\begin{aligned}
& c u_{X}^{\prime}=d u_{X}^{\prime \prime}+\mu-\mu u_{X}-\beta g\left(u_{X}\right) \varphi_{2}, \\
& c v_{X}^{\prime}=D_{v} v_{X}^{\prime \prime}+\beta g(\chi) \varphi_{2}+r \varphi_{2}-\sigma v_{X}, \\
& c w_{X}^{\prime}=D w_{X}^{\prime \prime}+\gamma \varphi_{1}-\delta w_{X}
\end{aligned}
$$

with

$$
u_{X}( \pm X)=\widetilde{F}( \pm X), \quad v_{X}( \pm X)=h_{1}( \pm X), \quad w_{X}( \pm X)=h_{2}( \pm X)
$$

for any $\left(\chi(\cdot), \varphi_{1}(\cdot), \varphi_{2}(\cdot)\right) \in \Gamma_{X}$. It follows from Gilbarg and Trudinger [11, Corollary 9.18] that the problem $3.7-3.10$ can admit a unique solution $\left(u_{X}, v_{X}, w_{X}\right)\left(u_{X}, v_{X}, w_{X} \in\right.$ 
$\left.W^{2, p}((-X, X), \mathbb{R}) \cap C([-X, X], \mathbb{R}), \forall p>1\right)$. Furthermore, according to the embedding theorem (see Gilbarg and Trudinger [11, Theorem 7.26]), it has $u_{X}(\cdot), v_{X}(\cdot), w_{X}(\cdot) \in$ $W^{2, p}(-X, X) \hookrightarrow C^{1+\alpha}[-X, X]$ for some $\alpha \in(0,1)$. Then we define an operator $T=$ $\left(T_{1}, T_{2}, T_{3}\right): \Gamma_{X} \rightarrow C\left([-X, X], \mathbb{R}^{3}\right)$ by

$$
u_{X}=T_{1}\left(\chi, \varphi_{1}, \varphi_{2}\right), \quad v_{X}=T_{2}\left(\chi, \varphi_{1}, \varphi_{2}\right), \quad w_{X}=T_{3}\left(\chi, \varphi_{1}, \varphi_{2}\right),
$$

for all $\left(\chi(\cdot), \varphi_{1}(\cdot), \varphi_{2}(\cdot)\right) \in \Gamma_{X}$.

Theorem 3.4. The operator $T$ maps $\Gamma_{X}$ into $\Gamma_{X}$.

Proof. It is obvious that 1 is a super-solution of $(3.7)$ for $\xi \in[-X, X]$. In addition, using Lemma 3.2 , we can see that $\widetilde{F}(\xi)=1-K e^{\theta \xi}$ satisfies

$$
\begin{aligned}
0 & >c \widetilde{F}^{\prime}(\xi)-d \widetilde{F}^{\prime \prime}(\xi)-\mu+\mu \widetilde{F}(\xi)+\beta \widetilde{M}_{2}(\xi) g(\widetilde{F}(\xi)) \\
& \geq c \widetilde{F}^{\prime}(\xi)-d \widetilde{F}^{\prime \prime}(\xi)-\mu+\mu \widetilde{F}(\xi)+\beta \varphi_{2} g(\widetilde{F}(\xi))
\end{aligned}
$$

for $\left[-X, X^{\prime}\right]$ with $X^{\prime}=-\frac{1}{\theta} \ln K$. It follows from the maximum principle associated with $u_{X}(-X)=\widetilde{F}(-X)$ and $u_{X}\left(X^{\prime}\right) \geq \widetilde{F}\left(X^{\prime}\right)=0$ that $\widetilde{F}(\xi) \leq u_{X}(\xi), \forall \xi \in\left[-X, X^{\prime}\right]$. As a consequence, we get $\widetilde{F}(\xi) \leq u_{X}(\xi) \leq 1$ for $\xi \in[-X, X]$.

Due to 3.1 and 3.2 , it has

$$
\begin{aligned}
0 & =-D_{v} \widetilde{M}_{1}^{\prime \prime}+c \widetilde{M_{1}^{\prime}}-\beta g(1) \widetilde{M}_{2}-r \widetilde{M}_{2}+\sigma \widetilde{M}_{1} \\
& \leq-D_{v} \widetilde{M}_{1}^{\prime \prime}+c \widetilde{M}_{1}^{\prime}-\beta \varphi_{2} g(\chi)-r \varphi_{2}+\sigma \widetilde{M}_{1}
\end{aligned}
$$

and

$$
0=-D \widetilde{M}_{2}^{\prime \prime}+c \widetilde{M_{2}^{\prime}}-\gamma \widetilde{M}_{1}+\delta \widetilde{M}_{2} \leq-D \widetilde{M}_{2}^{\prime \prime}+c \widetilde{M_{2}^{\prime}}-\gamma \varphi_{1}+\delta \widetilde{M_{2}}
$$

for any $\xi \in[-X, X]$. Thus, it has $v_{X}(\xi) \leq \widetilde{M}_{1}(\xi)$ and $w_{X}(\xi) \leq \widetilde{M}_{2}(\xi)$ for $\xi \in[-X, X]$ by the maximum principle. On the other hand, (3.4) and (3.5) imply that

$$
\begin{aligned}
0 & \geq-D_{v} h_{1}^{\prime \prime}(\xi)+c h_{1}^{\prime}(\xi)-\beta h_{2}(\xi) g(\widetilde{F}(\xi))-r h_{2}(\xi)+\sigma h_{1}(\xi) \\
& \geq-D_{v} h_{1}^{\prime \prime}(\xi)+c h_{1}^{\prime}(\xi)-\beta \varphi_{2}(\xi) g(\chi(\xi))-r \varphi_{2}(\xi)+\sigma h_{1}(\xi)
\end{aligned}
$$

for $\xi \in\left[-X, X_{1}^{\prime}\right]$ with $X_{1}^{\prime}=\frac{1}{\omega} \ln \frac{p}{B \zeta_{1}}$ and

$$
\begin{aligned}
0 & \geq-D h_{2}^{\prime \prime}(\xi)+c h_{2}^{\prime}(\xi)-\gamma h_{1}(\xi)+\delta h_{2}(\xi) \\
& \geq-D h_{2}^{\prime \prime}(\xi)+c h_{2}^{\prime}(\xi)-\gamma \varphi_{1}(\xi)+\delta h_{2}(\xi)
\end{aligned}
$$

for $\xi \in\left[-X, X_{2}^{\prime}\right]$ with $X_{2}^{\prime}=\frac{1}{\omega} \ln \frac{q}{B \zeta_{2}}$, respectively. According to $v_{X}(-X)=h_{1}(-X)$ and $v_{X}\left(X_{1}^{\prime}\right) \geq h_{1}\left(X_{1}^{\prime}\right)=0$, we get $v_{X}(\xi) \geq h_{1}(\xi)$ for $\xi \in\left[-X, X_{1}^{\prime}\right]$ by using the maximum principle. Similarly, on the basis of $w_{X}(-X)=h_{2}(-X)$ and $w_{X}\left(X_{2}^{\prime}\right) \geq h_{2}\left(X_{2}^{\prime}\right)=0$, it has $w_{X}(\xi) \geq h_{2}(\xi)$ for $\xi \in\left[-X, X_{2}^{\prime}\right]$. Therefore, one has $h_{1}(\xi) \leq v_{X}(\xi) \leq \widetilde{M}_{1}(\xi)$ and $h_{2}(\xi) \leq w_{X}(\xi) \leq \widetilde{M}_{2}(\xi)$ for $\xi \in[-X, X]$. This completes the proof. 
It follows from the classic embedding theorem that $T$ is a compact operator from $\Gamma_{X}$ to $\Gamma_{X}$. In fact, $T: \Gamma_{X} \rightarrow \Gamma_{X}$ is also a completely continuous operator [39]. As a consequence, using the Schauder's fixed point theorem, we can obtain that there exists a vector function $\left(u_{X}, v_{X}, w_{X}\right) \in \Gamma_{X}$ such that

$$
\left(u_{X}, v_{X}, w_{X}\right)=T\left(u_{X}, v_{X}, w_{X}\right)
$$

for $\xi \in[-X, X]$, namely,

$$
\begin{aligned}
& c u_{X}^{\prime}=d u_{X}^{\prime \prime}+\mu-\mu u_{X}-\beta w_{X} g\left(u_{X}\right), \\
& c v_{X}^{\prime}=D_{v} v_{X}^{\prime \prime}+\beta w_{X} g\left(u_{X}\right)+r w_{X}-\sigma v_{X}, \\
& c w_{X}^{\prime}=D w_{X}^{\prime \prime}+\gamma v_{X}-\delta w_{X}
\end{aligned}
$$

with $u_{X}( \pm X)=\widetilde{F}( \pm X), v_{X}( \pm X)=h_{1}( \pm X)$ and $w_{X}( \pm X)=h_{2}( \pm X)$.

Is the above conclusion true for $X \rightarrow \infty$ ? Next, we prove that the conclusion for $X \rightarrow \infty$ also holds true.

Theorem 3.5. For a given $Y>0$, there exist some positive constants $\mathcal{M}_{u}(Y), \mathcal{M}_{v}(Y)$ and $\mathcal{M}_{w}(Y)$, which are independent upon $X>\max \left\{Y, \mathcal{J}, \frac{1}{\theta} \ln K\right\}$, such that

$$
\left\|u_{X}\right\|_{C^{3}[-Y, Y]} \leq \mathcal{M}_{u}(Y), \quad\left\|v_{X}\right\|_{C^{3}[-Y, Y]} \leq \mathcal{M}_{v}(Y), \quad\left\|w_{X}\right\|_{C^{3}[-Y, Y]} \leq \mathcal{M}_{w}(Y)
$$

Proof. Note that

$$
u_{X}(\xi) \leq 1, \quad v(\xi) \leq p e^{\lambda_{c} Y}:=\widetilde{M}_{1}(Y), \quad w(\xi) \leq q e^{\lambda_{c} Y}:=\widetilde{M}_{2}(Y)
$$

for $\xi \in[-Y, Y]$. Using 3.15 and the $L^{p}(p \geq 2)$ estimates of linear elliptic differential equations to (3.11), it has

$$
\left\|u_{X}\right\|_{W^{2, p}(-Y, Y)} \leq \mathcal{O}\left(\mu+\beta g(1) \widetilde{M}_{2}(Y)+\|\chi\|_{W^{2, p}(-Y, Y)}\right)
$$

where $\mathcal{O}$ is a constant and depends on $Y, \chi$ can be treated as a linear function connecting the points $\left(-Y, u_{X}(-Y)\right)$ and $\left(Y, u_{X}(Y)\right)$. Thus, there exists a constant $\widehat{\mathcal{Q}}$ depending upon $Y$ such that $\left\|u_{X}\right\|_{W^{2, p}(-Y, Y)} \leq \widehat{\mathcal{Q}}(Y)$ for any $X>Y$. On the basis of $W^{2, p}(-Y, Y) \hookrightarrow C^{1, \alpha}[-Y, Y]$ for $\alpha=1-1 / p$, it has that a constant $M_{u 1}^{0}$ depending on $Y$ satisfies $\left\|u_{X}\right\|_{C^{1, \alpha}[-Y, Y]} \leq \mathcal{M}_{u 1}^{0}\left\|u_{X}\right\|_{W^{2, p}(-Y, Y)}$, which leads to $\left\|u_{X}\right\|_{C^{1, \alpha}[-Y, Y]} \leq \widetilde{M}_{u}(Y)$ for $\widetilde{M}_{u}(Y)=\mathcal{M}_{u 1}^{0} \widehat{\mathcal{Q}}(Y)>0$. Due to (3.11), we further get that $\left\|u_{X}\right\|_{C^{2}[-Y, Y]} \leq \widetilde{M}_{u}(Y)$ for some $\widetilde{M}_{u}(Y)>0$. Using the same way, $\left\|v_{X}\right\|_{C^{2}[-Y, Y]} \leq \widetilde{M}_{v}(Y)$ and $\left\|w_{X}\right\|_{C^{2}[-Y, Y]} \leq$ $\widetilde{M}_{w}(Y)$ for some constants $\widetilde{M}_{v}(Y)>0$ and $\widetilde{M}_{w}(Y)>0$ can be obtained. By differentiating two sides of the equations 3.11 $-(3.13)$, it further gets the estimates 3.14). This completes the proof. 
Next, consider a sequence of positive numbers $\left\{X_{m}\right\}_{m>0}$ such that $X_{m} \rightarrow+\infty$ as $m \rightarrow+\infty$. In view of the above arguments, it is easy to see that there exists a solution $(u, v, w) \in C^{2}\left(\mathbb{R}, \mathbb{R}^{3}\right)$ of 2.4 such that

$$
\widetilde{F}(x) \leq u(x) \leq 1, \quad h_{1}(x) \leq v(x) \leq \widetilde{M}_{1}(x), \quad h_{2}(x) \leq w(x) \leq \widetilde{M}_{2}(x) .
$$

In particular, the definitions of $\widetilde{F}(x), h_{i}(x)$ and $\widetilde{M}_{i}(x)$ imply that

$$
u(x) \rightarrow 1, \quad \frac{1}{p} e^{-\lambda_{c} x} v(x) \rightarrow 1, \quad \frac{1}{q} e^{-\lambda_{c} x} w(x) \rightarrow 1
$$

as $x \rightarrow-\infty$, which means that

$$
\lim _{x \rightarrow \infty} u(x)=1, \quad \lim _{x \rightarrow \infty} v(x)=0, \quad \lim _{x \rightarrow \infty} w(x)=0 .
$$

Finally, we show

$$
u(x) \rightarrow u^{*}, \quad v(x) \rightarrow v^{*}, \quad w(x) \rightarrow w^{*} \quad \text { as } x \rightarrow+\infty .
$$

For convenience, let $\sigma=\gamma+\mu_{1}, \delta=r+\mu_{2}$ and $\widehat{\mu}=\min \left\{\mu, \mu_{1}, \mu_{2}\right\}$.

Lemma 3.6. Let (A) be satisfied. Then these following inequalities

$$
0<v(x)+w(x) \leq \frac{\mu}{\widehat{\mu}} \sqrt{\frac{d}{D_{\min }}}
$$

and

$$
\frac{\mu}{\mu+\beta g\left(\frac{\mu}{\widehat{\mu}} \sqrt{\frac{d}{D_{\text {min }}}}\right)} \leq u(x) \leq 1, \quad x \in \mathbb{R}
$$

hold true, where $D_{\min }=\min \left\{D_{v}, D\right\}$.

Proof. Note that $D_{\text {min }}$ is defined as above. Let $m(x)=\beta w(x) g(u(x))$ and $n(x)=\gamma v(x)-$ $r w(x)$ for any $x \in \mathbb{R}$, then it has

$$
\begin{aligned}
-d u^{\prime \prime}(x)+c u^{\prime}(x)+\widehat{\mu} u(x) & \leq \mu-m(x), \\
-D_{v} v^{\prime \prime}(x)+c v^{\prime}(x)+\widehat{\mu} v(x) & \leq m(x)-n(x), \\
-D w^{\prime \prime}(x)+c w^{\prime}(x)+\widehat{\mu} w(x) & \leq n(x) .
\end{aligned}
$$

As a consequence, we can obtain

$$
\begin{aligned}
u(x) & \leq \frac{\mu}{\widehat{\mu}}-f_{d}(x), \quad x \in \mathbb{R}, \\
v(x) & \leq f_{D_{v}}(x)-g_{D_{v}}(x), \quad x \in \mathbb{R}, \\
w(x) & \leq g_{D}(x), \quad x \in \mathbb{R},
\end{aligned}
$$


where

$$
f_{d}(x)=\int_{0}^{+\infty} \frac{e^{-\widehat{\mu} t}}{\sqrt{4 \pi d t}} \int_{-\infty}^{+\infty} m(y-x-c t) e^{-y^{2} /(4 d t)} d y d t
$$

and

$$
g_{D}(x)=\int_{0}^{+\infty} \frac{e^{-\widehat{\mu} t}}{\sqrt{4 \pi D t}} \int_{-\infty}^{+\infty} n(y-x-c t) e^{-y^{2} /(4 D t)} d y d t
$$

It follows from $d \geq D_{v} \geq D$ that $\sqrt{D_{v}} g_{D_{v}}(x) \geq \sqrt{D} g_{D}(x)$ and $\sqrt{d} f_{d}(x) \geq \sqrt{D_{v}} f_{D_{v}}(x)$ for any $x \in \mathbb{R}$, which further indicates that

$$
\sqrt{D} w(x) \leq \sqrt{D} g_{D}(x), \quad \forall x \in \mathbb{R}
$$

and

$$
v(x)+\sqrt{\frac{D}{D_{v}}} w(x) \leq f_{D_{v}}(x) \leq \sqrt{\frac{d}{D_{v}}} f_{d}(x) \leq \sqrt{\frac{d}{D_{v}}}\left(\frac{\mu}{\widehat{\mu}}-S(x)\right) \leq \frac{\mu}{\widehat{\mu}} \sqrt{\frac{d}{D_{v}}}, \quad \forall x \in \mathbb{R} .
$$

Thus, we get

$$
\sqrt{D_{v}} v(x)+\sqrt{D} w(x) \leq \frac{\mu \sqrt{d}}{\widehat{\mu}}, \quad \forall x \in \mathbb{R}
$$

which implies that

$$
v(x)+w(x) \leq \sqrt{\frac{D_{v}}{D_{\min }}} v(x)+\sqrt{\frac{D}{D_{\min }}} w(x) \leq \frac{\mu}{\widehat{\mu}} \sqrt{\frac{d}{D_{\min }}}, \quad \forall x \in \mathbb{R} .
$$

Besides, $u(x)$ satisfies the following inequality

$$
d u^{\prime \prime}(x)-c u^{\prime}(x)+\mu-\left(\mu+\beta g\left(\frac{\mu}{\widehat{\mu}} \sqrt{\frac{d}{D_{\min }}}\right)\right) u(x) \leq 0, \quad \forall x \in \mathbb{R} .
$$

Using the maximum principle, it has that

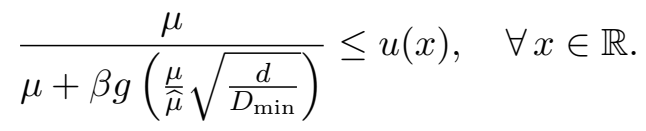

The proof is completed.

Then, similar to [39, Section 2], we get that there exists an $M_{1}>0$ satisfying for any $x \in \mathbb{R}$,

$$
\max \left\{\max _{[x-1, x+1]} v, \max _{[x-1, x+1]} w\right\} \leq M_{1} \min \left\{\min _{[x-1, x+1]} v, \underset{[x-1, x+1]}{\min } w\right\}
$$

and there exists a positive constant $\bar{M}$ satisfying

$$
\left|\frac{v^{\prime}(x)}{v(x)}\right|+\left|\frac{w^{\prime}(x)}{w(x)}\right| \leq \bar{M}, \quad \forall x \in \mathbb{R} .
$$


Now, we use some suitable Lyapunov functional to prove the convergence of traveling waves as $x \rightarrow+\infty$. Let $\mathcal{L}(x)=x-1-\ln x$. Define

$$
\overline{\mathcal{C}}=\left\{\begin{array}{c}
(u(\cdot), v(\cdot), w(\cdot)) \in C^{1}(\mathbb{R},(0,+\infty)) \times C^{1}(\mathbb{R},(0,+\infty)) \times C^{1}(\mathbb{R},(0,+\infty)): \\
u>0, v>0, w>0, \exists \bar{M}>0,\left|\frac{v^{\prime}(x)}{v(x)}\right|+\left|\frac{w^{\prime}(x)}{w(x)}\right| \leq \bar{M}
\end{array}\right\} .
$$

For each $(u, v, w) \in \overline{\mathcal{C}}$, define

$$
\begin{aligned}
V(u, v, w)(x):= & {\left[d u^{\prime}(x)\left(\frac{g\left(u^{*}\right)}{g(u(x))}-1\right)+c \int_{u^{*}}^{u(x)} \frac{g(\xi)-g\left(u^{*}\right)}{g(\xi)} d \xi\right] } \\
& +\left[D v^{\prime}(x)\left(\frac{1}{v(x)}-\frac{1}{v^{*}}\right)+c \mathcal{L}\left(\frac{v(x)}{v^{*}}\right)\right] v^{*} \\
& +C\left[D w^{\prime}(x)\left(\frac{1}{w(x)}-\frac{1}{w^{*}}\right)+c \mathcal{L}\left(\frac{w(x)}{w^{*}}\right)\right] w^{*}
\end{aligned}
$$

where

$$
C=\frac{\beta g\left(u^{*}\right) w^{*}+r w^{*}}{\delta w^{*}}
$$

Then the following result is proved.

Theorem 3.7. Let (H1) be satisfied. System (2.4) admits a positive solution $(u(\cdot), v(\cdot)$, $w(\cdot))$ satisfying there exists a $\mathcal{P}>1$ such that

$$
\frac{1}{\mathcal{P}} \leq u(x) \leq 1, \quad 0<w(x) \leq \mathcal{P} w^{*}, \quad 0<v(x) \leq \mathcal{P} v^{*}
$$

and

$$
\left|\frac{v^{\prime}(x)}{v(x)}\right|+\left|\frac{w^{\prime}(x)}{w(x)}\right| \leq \mathcal{P}
$$

for any $x \in \mathbb{R}$. Thus there exists a positive constant $m$ satisfying

$$
-m \leq V(x)<\infty, \quad \forall x \in \mathbb{R}
$$

and the map $x \rightarrow V(x)$ is non-increasing. Especially, if $x \rightarrow V(x)$ is a constant, then $u \equiv u^{*}, v \equiv v^{*}, w \equiv w^{*}$.

Proof. According to the inequalities 3.19-3.20), for any $x \in \mathbb{R}$, we have

$$
\begin{aligned}
& \left|d u^{\prime}(x)\left(\frac{g\left(u^{*}\right)}{g(u(x))}-1\right)+D_{v} v^{\prime}(x)\left(\frac{1}{v(x)}-\frac{1}{v^{*}}\right)+C D w^{\prime}(x)\left(\frac{1}{w(x)}-\frac{1}{w^{*}}\right)\right| \\
\leq & d \mathcal{M}\left\|u^{\prime}\right\|_{\infty}\left(1+\frac{g\left(u^{*}\right)}{g\left(\mathcal{M} u^{*}\right)}\right)+\widetilde{D} \bar{C} \mathcal{M}+\widetilde{D} \bar{C}\left(\left|\frac{v^{\prime}(x)}{v^{*}}\right|+\left|\frac{w^{\prime}(x)}{w^{*}}\right|\right) \\
\leq & d \mathcal{M}\left\|u^{\prime}\right\|_{\infty}\left(1+\frac{g\left(u^{*}\right)}{g\left(\mathcal{M} u^{*}\right)}\right)+\widetilde{D} \bar{C} \mathcal{M}+\widetilde{D} \bar{C} \mathcal{M}\left(\left|\frac{v^{\prime}(x)}{v(x)}\right|+\left|\frac{w^{\prime}(x)}{w(x)}\right|\right) \\
\leq & d \mathcal{M}\left\|u^{\prime}\right\|_{\infty}\left(1+\frac{g\left(u^{*}\right)}{g\left(\mathcal{M} u^{*}\right)}\right)+\widetilde{D} \bar{C} \mathcal{M}+\widetilde{D} \bar{C} \mathcal{M}^{2},
\end{aligned}
$$


where $\widetilde{D}=\max \left\{D_{v}, C D\right\}$ and $\bar{C}=\max \{1, C\}$. Set

$$
\omega(x)=c \int_{u^{*}}^{u(x)} \frac{g(\xi)-g\left(u^{*}\right)}{g(\xi)} d \xi+c \mathcal{L}\left(\frac{v(x)}{v^{*}}\right)+c C \mathcal{L}\left(\frac{w(x)}{w^{*}}\right), \quad x \in \mathbb{R} .
$$

Based on the definition of the function $\mathcal{L}$ and the inequalities $(3.19)$, it gets

$$
0 \leq \omega(x)<+\infty, \quad x \in \mathbb{R}
$$

From 3.22 and the last inequality, it follows that inequality 3.21 holds.

By a direct calculation, we get

$$
\begin{aligned}
\frac{d V(x)}{d x}= & \mu-\beta g(u) w-\mu u-\mu \frac{g\left(u^{*}\right)}{g(u)}+\beta g\left(u^{*}\right) w+\mu u^{*} \frac{g\left(u^{*}\right)}{g(u(x))}-\frac{d g^{\prime}(u(x))\left(u^{\prime}(x)\right)^{2}}{g^{2}(u(x))} \\
& +\beta g(u) w+r w-\sigma v-\beta g(u) w \frac{v^{*}}{v}+\sigma v^{*}-r w \frac{v^{*}}{v}-\frac{v^{\prime 2}(x) v^{*}}{v^{2}(x)} \\
& +C\left(\gamma v-\delta w-\gamma v \frac{w^{*}}{w}+\delta w^{*}-\frac{w^{\prime 2}(x) w^{*}}{w^{2}(x)}\right) .
\end{aligned}
$$

On the basis of the following equalities

$$
\mu=\beta g\left(u^{*}\right) w^{*}+\mu u^{*}, \quad \sigma v^{*}=\beta g\left(u^{*}\right) w^{*}+r w^{*} \quad \text { and } \quad \gamma v^{*}=\delta w^{*},
$$

it gets

$$
\begin{aligned}
\frac{d V}{d x}= & \mu\left(u^{*}-u\right) \frac{g(u(x))-g\left(u^{*}\right)}{g(u(x))}+\beta g\left(u^{*}\right) w^{*}\left(3-\frac{g\left(u^{*}\right)}{g(u(x))}-\frac{g(u(x)) v^{*} w}{g\left(u^{*}\right) v w^{*}}-\frac{v w^{*}}{v w^{*}}\right) \\
& +r w^{*}\left(2-\frac{v w^{*}}{v^{*} w}-\frac{v^{*} w}{v w^{*}}\right)-\frac{d g^{\prime}(u(x))\left(u^{\prime}(x)\right)^{2}}{g^{2}(u(x))}-\frac{v^{\prime 2}(x) v^{*}}{v^{2}(x)}-C \frac{w^{\prime 2}(x) w^{*}}{w^{2}(x)} .
\end{aligned}
$$

Due to the mean inequality, we know that

$$
\frac{g\left(u^{*}\right)}{g(u(x))}+\frac{g(u(x)) v^{*} w}{g\left(u^{*}\right) v w^{*}}+\frac{v w^{*}}{v^{*} w} \geq 3, \quad \frac{v^{*} w}{v w^{*}}+\frac{v w^{*}}{v^{*} w} \geq 2, \quad \forall x \in \mathbb{R} .
$$

In addition, it follows from the second assumption of (H1) that

$$
\left(u^{*}-u\right) \frac{g(u(x))-g\left(u^{*}\right)}{g(u(x))} \leq 0, \quad \forall u \geq 0 .
$$

As a consequence, it has $d V / d x \leq 0, \forall x \in \mathbb{R}$, precisely, the function is non-increasing in $x \in \mathbb{R}$. When $V(x)$ is a constant, we obtain

$$
\frac{d V(x)}{d x} \equiv 0, \quad \forall x \in \mathbb{R}
$$

which states that

$(3.23) \quad u^{\prime}(x) \equiv 0, \quad v^{\prime}(x) \equiv 0, \quad w^{\prime}(x) \equiv 0, \quad u(x) \equiv u^{*}, \quad v^{*} w(x) \equiv w^{*} v(x), \quad \forall x \in \mathbb{R}$. 
It further follows from 3.23 that

$$
u(x) \equiv u^{*}, \quad v(x) \equiv A, \quad w(x) \equiv B, \quad \forall x \in \mathbb{R},
$$

where $A$ and $B$ are two nonnegative constants. Note that $(u, v, w)$ is a solution of system (2.4). Thus, plugging (3.24) into system (2.4), it has

$$
u(x) \equiv u^{*}, \quad v(x) \equiv v^{*}, \quad w(x) \equiv w^{*}, \quad \forall x \in \mathbb{R} .
$$

This completes this proof.

Based on the previous arguments, it has that there exist functions

$$
(u(\cdot), v(\cdot), w(\cdot))
$$

such that (2.4), (3.16), 3.17), 3.18) and

$$
\lim _{x \rightarrow-\infty} u(x)=1, \quad \lim _{x \rightarrow-\infty} v(x)=\lim _{x \rightarrow-\infty} w(x)=0 .
$$

Consider an increasing sequence $\left\{x_{m}\right\}_{m \geq 0}$ which owns positive real numbers so that $x_{m} \rightarrow$ $+\infty$ as $m \rightarrow+\infty$. For $\forall m \in \mathbb{N}$, define

$$
u_{m}(x)=u\left(x+x_{m}\right), \quad v_{m}(x)=v\left(x+x_{m}\right), \quad w_{m}(x)=w\left(x+x_{m}\right), \quad x \in \mathbb{R} .
$$

According to elliptic estimates, up to a sequence, assume that the sequences $\left\{u_{m}\right\}$, $\left\{v_{m}\right\}$ and $\left\{w_{m}\right\}$ converge towards some functions $u_{\infty}, v_{\infty}, w_{\infty}$ in $C_{\text {loc }}^{2}(\mathbb{R}) \times C_{\text {loc }}^{2}(\mathbb{R}) \times$ $C_{\text {loc }}^{2}(\mathbb{R})$. Consequently, system (2.4)-2.5 admits solutions $\left(u_{\infty}, v_{\infty}, w_{\infty}\right)$. Due to the non-increasing of the map $x \rightarrow V(x)$, for each $m \geq 0$, it has

$$
\begin{aligned}
V\left(u_{m}, v_{m}, w_{m}\right)(x) & =V(u, v, w)\left(x+x_{m}\right) \\
& \leq V(u, v, w)(x), \quad \forall x \in \mathbb{R} .
\end{aligned}
$$

On the basis of the uniform boundedness of the sequence $\left\{V\left(u_{m}, v_{m}, w_{m}\right)(x)\right\}_{m \in \mathbb{N}}$, there exists $l \in \mathbb{R}$ satisfying

$$
\lim _{m \rightarrow+\infty} V\left(u_{m}, v_{m}, w_{m}\right)(x)=l, \quad \forall x \in \mathbb{R} .
$$

According to

$$
\lim _{m \rightarrow+\infty} V\left(u_{m}, v_{m}, w_{m}\right)(x)=V\left(u_{\infty}, v_{\infty}, w_{\infty}\right)(x)
$$

for $x \in C_{\text {loc }}^{1}(\mathbb{R})$, one gets $V\left(u_{\infty}, v_{\infty}, w_{\infty}\right)(x) \equiv l$. Theorem 3.7 indicates that

$$
u_{\infty} \equiv u^{*}, \quad v_{\infty} \equiv v^{*}, \quad w_{\infty} \equiv w^{*} .
$$

Then it follows that

$$
\lim _{x \rightarrow+\infty} u(x)=u^{*}, \quad \lim _{x \rightarrow+\infty} v(x)=v^{*}, \quad \lim _{x \rightarrow+\infty} w(x)=w^{*} .
$$

This completes the proof of the existence of traveling wave solutions of system (1.1) if $R_{0}>1$ and $c>c^{*}$. 


\subsection{Case II: $R_{0}>1$ and $c=c^{*}$}

Secondly, we show that if $R_{0}>1$ and $c=c^{*}$, then system 1.1) admits a traveling wave solution $(u(\xi), v(\xi), w(\xi))$ on $\mathbb{R}^{3}$ such that (2.4) and 2.5). We prove the conclusion by using the similar argument as those in [39, Theorem 2.11]. Thus, we omit it.

\section{Proof of assertion (ii) in Theorem 2.3}

In the section, we show the nonexistence of nonnegative traveling wave solutions of system (1.1) when $R_{0}>1$ and $0<c<c^{*}$. We first discuss some properties of $v(x)$ and $w(x)$.

Lemma 4.1. Assume $R_{0}>1$. For any $c>0$, if there exists a positive traveling wave solution $(u(x+c t), v(x+c t), w(x+c t))$ of system (1.1) such that (2.4) and (2.5), then there are two positive constants $\mathcal{T}$ and $M$ which is large enough satisfying

$$
\int_{-\infty}^{x} v(\xi) d \xi \leq \mathcal{T} \quad \text { and } \quad \int_{-\infty}^{x} w(\xi) d \xi \leq \mathcal{T}, \quad x<-2 M
$$

Proof. Fix $c>0$. Let $(u(x+c t), v(x+c t), w(x+c t))$ be a nonnegative traveling waves of (1.1) which satisfies (2.4) and (2.5). According to $u(-\infty)=1$, there exists an $M>0$ large enough satisfying

$$
u(\xi)>(1-\nu), \quad \forall \xi \in(-\infty,-2 M),
$$

where $\nu \in(0,1)$ which is a small constant will be determined later.

For each $\xi<-2 M$, it has

$$
\beta g(u(\xi)) w(\xi)+r w(\xi)-\sigma v(\xi) \geq \beta g(1-\nu) w(\xi)+r w(\xi)-\sigma v(\xi) .
$$

For $y<x<-2 M$, set $\widetilde{J}_{v}(x, y)=\int_{y}^{x} v(\xi) d \xi$ and $\widetilde{J}_{w}(x, y)=\int_{y}^{x} w(\xi) d \xi$. In addition, we can get

$$
\beta g(1-\nu) \widetilde{J}_{w}(x, y)+r \widetilde{J}_{w}(x, y)-\sigma \widetilde{J}_{v}(x, y) \leq \int_{y}^{x}(\beta g(u(\xi)) w(\xi)+r w(\xi)-\sigma v(\xi)) d \xi
$$

by integrating two sides of 4.1) from $y$ to $x(y<x<-2 M)$.

Based on Lemma 3.6 and (3.18), we can obtain

$$
\|v(\cdot)\|_{C^{2}(\mathbb{R})},\|w(\cdot)\|_{C^{2}(\mathbb{R})} \leq \overline{\mathcal{P}}, \quad \lim _{x \rightarrow-\infty} v^{\prime}(x)=\lim _{x \rightarrow-\infty} w^{\prime}(x)=0
$$

where $\overline{\mathcal{P}}$ is a positive constant. From 4.3 , it follows that

$$
\int_{-\infty}^{x}(\beta g(u(\xi)) w(\xi)+r w(\xi)-\sigma v(\xi)) d \xi=-D w^{\prime}(x)+c w(x), \quad \forall x \in(-\infty,+\infty)
$$


and

$$
\int_{-\infty}^{x}(\gamma v(\xi)-\delta w(\xi)) d \xi=-D_{v} v^{\prime}(x)+c v(x), \quad \forall x \in(-\infty,+\infty) .
$$

Now, we prove that there exists a positive constant $\mathcal{T}>0$ so that

$$
\int_{-\infty}^{x} v(\xi) d \xi \leq \mathcal{T} \quad \text { and } \quad \int_{-\infty}^{x} w(\xi) d \xi \leq \mathcal{T}, \quad \forall x<-2 M .
$$

Let

$$
\mathcal{A}=\left[\begin{array}{cc}
-\sigma & \beta g(1-\nu)+r \\
\gamma & -\delta
\end{array}\right]
$$

On the basis of $R_{0}=(\beta g(1)+r) \gamma /(\sigma \delta)>1$, it gets $|\mathcal{A}|<0$ for $\nu>0$ sufficiently small. Using multiplying 4.2 by $-\delta$ and then adding

$$
-(\beta g(1-\nu)+r)\left(\gamma \widetilde{J}_{v}(x, y)-\delta \widetilde{J}_{w}(x, y)\right)
$$

to the both sides of the inequality, it obtains

$$
\begin{aligned}
-|\mathcal{A}| \widetilde{J}_{v}(x, y) d \xi \leq & \delta \int_{y}^{x}(\beta g(u(\xi)) w(\xi)+r w(\xi)-\sigma v(\xi)) d \xi \\
& +(\beta g(1-\nu)+r)\left(\gamma \widetilde{J}_{v}(x, y)-\delta \widetilde{J}_{w}(x, y)\right)
\end{aligned}
$$

where $y<x<-2 M$. Letting $y \rightarrow-\infty$ in (4.6) and then using (4.4) and (4.5), it gets

$$
\int_{-\infty}^{x} v(\xi) d \xi \leq \mathcal{T}, \quad \forall x<-2 M
$$

In the same method, we can show that

$$
\int_{-\infty}^{x} w(\xi) d \xi \leq \mathcal{T}, \quad \forall x<-2 M
$$

The proof is completed.

Lemma 4.2. Assume $R_{0}>1$. For any $c>0$, if system 1.1 admits a nontrivial and nonnegative traveling wave solution $(u(x+c t), v(x+c t), w(x+c t))$ which satisfies (2.5), then there is a positive constant $\mu_{0}$ such that

$$
\sup _{x \in \mathbb{R}}\left\{v(x) e^{-\mu_{0} x}\right\}<+\infty, \quad \sup _{x \in \mathbb{R}}\left\{\left|v^{\prime}(x)\right| e^{-\mu_{0} x}\right\}<+\infty, \quad \sup _{x \in \mathbb{R}}\left\{\left|v^{\prime \prime}(x)\right| e^{-\mu_{0} x}\right\}<+\infty
$$

and

$$
\sup _{x \in \mathbb{R}}\left\{w(x) e^{-\mu_{0} x}\right\}<+\infty, \quad \sup _{x \in \mathbb{R}}\left\{\left|w^{\prime}(x)\right| e^{-\mu_{0} x}\right\}<+\infty, \quad \sup _{x \in \mathbb{R}}\left\{\left|w^{\prime \prime}(x)\right| e^{-\mu_{0} x}\right\}<+\infty
$$


Proof. Fix $c>0$. Assume that $(u(x+c t), v(x+c t), w(x+c t))$ is a nontrivial traveling wave solution of system (2.4)-2.5). It follows from $u(-\infty)=1$ and $u(\xi) \leq 1, \forall \xi \in \mathbb{R}$ that there exists $M_{\nu}>0$ sufficiently large such that

$$
u(\xi)>1-\nu, \quad \forall \xi \in\left(-\infty,-2 M_{\nu}\right)
$$

and

$$
\frac{(\beta g(1-\nu)+r) \gamma}{\sigma \delta}>1 \quad\left(R_{0}>1\right)
$$

where $\nu \in(0,1)$ is a small constant and then will be determined later. For $\forall \xi<-2 M_{\nu}$, it has

$$
c v^{\prime}(\xi) \geq D_{v} v^{\prime \prime}(\xi)+(\beta g(1-\nu)+r) w(\xi)-\sigma v(\xi)
$$

and

$$
c w^{\prime}(\xi)=D w^{\prime \prime}(\xi)-\gamma E(\xi)-\delta w(\xi)
$$

Due to Lemma 4.1, it has $J_{0}(\xi):=\int_{-\infty}^{\xi} v(\eta) d \eta<+\infty$ and $J_{1}(\xi):=\int_{-\infty}^{0} w(\eta) d \eta<+\infty$ for $\xi \leq 0$. Integrating two sides of inequality (4.8) from $-\infty$ to $\xi$ with $\xi<-2 M_{\nu}$, it has

$$
c v(\xi) \geq D_{v} v^{\prime}(\xi)+(\beta g(1-\nu)+r) J_{1}(\xi)-\sigma J_{0}(\xi)
$$

Furthermore, integrating two sides of inequality 4.9 from $-\infty$ to $\xi\left(\xi<-2 M_{\nu}\right)$ causes to

$$
(\beta g(1-\nu)+r) \int_{-\infty}^{\xi} J_{1}(x) d x-\sigma \int_{-\infty}^{\xi} J_{0}(x) d x+D_{v} v(\xi) \leq c J_{0}(\xi) .
$$

Similarly, for $\xi<-2 M_{\nu}$, we also obtain that

$$
\gamma \int_{-\infty}^{\xi} J_{0}(x) d x-\delta \int_{-\infty}^{\xi} J_{1}(x) d x+D w(\xi)=c J_{1}(\xi)
$$

which can reduce to

$$
\int_{-\infty}^{\xi} J_{1}(x) d x=\frac{1}{\delta}\left(\gamma \int_{-\infty}^{\xi} J_{0}(x) d x-c J_{1}(\xi)+D w(\xi)\right) .
$$

In the following, we prove that there are two positive constants $a, b$ such that

$$
a \sum_{i=0}^{1} \int_{-\infty}^{\xi} J_{i}(\eta) d \eta \leq b \sum_{i=0}^{1} J_{i}(\xi), \quad \forall \xi<-2 M_{\nu}
$$


Substituting 4.12 into 4.10, one has

$$
\begin{aligned}
c J_{0}(\xi) \geq & (\beta g(1-\nu)+r) \frac{\left(\gamma \int_{-\infty}^{\xi} J_{0}(x) d x-c J_{1}(\xi)+D w(\xi)\right)}{\delta} \\
& -\sigma \int_{-\infty}^{\xi} J_{0}(x) d x+D_{v} v(\xi) \\
= & \left(\frac{(\beta g(1-\nu)+r) \gamma}{\delta}-\sigma\right) \int_{-\infty}^{\xi} J_{0}(\eta) d \eta+\frac{(\beta g(1-\nu)+r) D}{\delta} w(\xi)+D_{v} v(\xi) \\
& -c \frac{\beta g(1-\nu)+r}{\delta} J_{1}(\xi),
\end{aligned}
$$

which implies that

$$
\begin{aligned}
c\left(J_{0}(\xi)+\frac{\beta g(1-\nu)+r}{\delta} J_{1}(\xi)\right) \geq & \left(\frac{(\beta g(1-\nu)+r) \gamma}{\delta}-\sigma\right) \int_{-\infty}^{\xi} J_{0}(\eta) d \eta \\
& +\frac{(\beta g(1-\nu)+r) D}{\delta} w(\xi)+D_{v} v(\xi) .
\end{aligned}
$$

Since $v(\xi)$ and $w(\xi)$ are positive functions, then one has

$$
\left(\frac{(\beta g(1-\nu)+r) \gamma}{\delta}-\sigma\right) \int_{-\infty}^{\xi} J_{0}(\eta) d \eta \leq c\left(J_{0}(\xi)+\frac{\beta g(1-\nu)+r}{\delta} J_{1}(\xi)\right) .
$$

Due to 4.7), it is obvious that

$$
\frac{(\beta g(1-\nu)+r) \gamma}{\delta}-\sigma>0
$$

As a consequence, there exists $\bar{a}_{0}>0$ and $\bar{b}_{0}>0$ such that

$$
\bar{a}_{0} \int_{-\infty}^{\xi} J_{0}(\eta) d \eta \leq \bar{b}_{0}\left(J_{0}(\xi)+J_{1}(\xi)\right), \quad \forall \xi<-2 M_{\nu}
$$

Plugging 4.14 into 4.10, we can obtain that there exists $\bar{a}>0$ and $\bar{b}>0$ such that

$$
\bar{a} \int_{-\infty}^{\xi} J_{1}(\eta) d \eta \leq \bar{b}\left(J_{0}(\xi)+J_{1}(\xi)\right), \quad \forall \xi<-2 M_{\nu}
$$

Adding 4.14 and 4.15, we can obtain the inequality 4.13).

Let

$$
\mathcal{D}(\xi)=J_{0}(\xi)+J_{1}(\xi)
$$

Then we have that

$$
a \int_{-\infty}^{\xi} \mathcal{D}(\eta) d \eta \leq b \mathcal{D}(\xi), \quad \forall \xi<-2 M_{\nu}
$$

which meas that

$$
a \int_{-\infty}^{0} \mathcal{D}(\xi+\eta) d \eta \leq b \mathcal{D}(\xi), \quad \forall \xi<-2 M_{\nu}
$$


$\mathcal{D}(\cdot)$ is increasing, so it has $a \eta \mathcal{D}(\xi-\eta) \leq b \mathcal{D}(\xi)$ for any $\xi<-2 M_{\nu}$ and any $\eta>0$. As a consequence, there exist an $\eta_{0}>0$ large enough and $\omega_{0} \in(0,1)$ satisfying

$$
\mathcal{D}\left(\xi-\eta_{0}\right) \leq \omega_{0} \mathcal{D}(\xi), \quad \forall \xi<-2 M_{\nu}
$$

Let $q(x)=\mathcal{D}(x) e^{-\mu_{0} x}$ with $0<\mu_{0}=\frac{1}{\eta_{0}} \ln \frac{1}{\omega_{0}}<\lambda_{c}$. Then, it gets

$$
q\left(\xi-\eta_{0}\right)=J\left(\xi-\eta_{0}\right) e^{-\mu_{0}\left(\xi-\eta_{0}\right)} \leq \omega_{0} J(\xi) e^{-\mu_{0}\left(\xi-\eta_{0}\right)}=q(\xi), \quad \xi<-2 M_{\nu}
$$

Since $\mathcal{D}(x)<+\infty$ for $x<0$, we have that there exists a constant $\kappa_{0}$ such that

$$
q(x) \leq \kappa_{0}, \quad \forall x<0,
$$

which implies that $\mathcal{D}(x) \leq \kappa_{0} e^{\mu_{0} x}$ for any $x<0$. Consequently, there exists $q_{0}>0$ satisfying $\int_{-\infty}^{x} J_{i}(\eta) d \eta \leq q_{0} e^{\mu_{0} x}$ for any $x<-2 M_{\nu}, i=0,1$. It follows from inequalities (4.10) and (4.11) that there exists $p_{0}>0$ such that

$$
v(x) \leq p_{0} e^{\mu_{0} x}, \quad w(x) \leq p_{0} e^{\mu_{0} x}, \quad \forall x<-2 M_{\nu}
$$

Since $v(x)$ and $w(x)$ are bounded on $x \in \mathbb{R}$, then we obtain

$$
v(x) \leq p_{0} e^{\mu_{0} x}, \quad w(x) \leq p_{0} e^{\mu_{0} x}, \quad \forall x \in \mathbb{R} .
$$

Using (4.9) and (3.18), we can get

$$
\sup _{x \in \mathbb{R}}\left\{\left|v^{\prime}(x)\right| e^{-\mu_{0} x}\right\}<+\infty
$$

Furthermore, by 4.8 , it has

$$
\sup _{x \in \mathbb{R}}\left\{\left|v^{\prime \prime}(x)\right| e^{-\mu_{0} x}\right\}<+\infty .
$$

Applying the same methods as $v(\xi)$, we also obtain

$$
\sup _{x \in \mathbb{R}}\left\{w(x) e^{-\mu_{0} x}\right\}<+\infty, \quad \sup _{x \in \mathbb{R}}\left\{\left|w^{\prime}(x)\right| e^{-\mu_{0} x}\right\}<+\infty, \quad \sup _{x \in \mathbb{R}}\left\{\left|w^{\prime \prime}(x)\right| e^{-\mu_{0} x}\right\}<+\infty .
$$

This completes the proof.

Now, we prove the main result of this subsection.

Theorem 4.3. Assume that $R_{0}>1$. For $c \in\left(0, c^{*}\right)$, there exists no nontrivial solution $(u(x+c t), v(x+c t), w(x+c t))$ of system (2.4)-2.5). 
Proof. We prove the theorem by way of contradiction. Fix $c \in\left(0, c^{*}\right)$. Suppose on the contrary that system (2.4) 2.5 admits a nontrivial solution $(u(x+c t), v(x+c t), w(x+c t))$. By Lemma 4.2, there are

$$
\sup _{x \in \mathbb{R}}\left\{v(x) e^{-\mu_{0} x}\right\}<+\infty, \quad \sup _{x \in \mathbb{R}}\left\{\left|v^{\prime}(x)\right| e^{-\mu_{0} x}\right\}<+\infty, \quad \sup _{x \in \mathbb{R}}\left\{\left|v^{\prime \prime}(x)\right| e^{-\mu_{0} x}\right\}<+\infty
$$

and

$$
\sup _{x \in \mathbb{R}}\left\{w(x) e^{-\mu_{0} x}\right\}<+\infty, \quad \sup _{x \in \mathbb{R}}\left\{\left|w^{\prime}(x)\right| e^{-\mu_{0} x}\right\}<+\infty, \quad \sup _{x \in \mathbb{R}}\left\{\left|w^{\prime \prime}(x)\right| e^{-\mu_{0} x}\right\}<+\infty .
$$

Set $R(\xi):=1-u(\xi) \in[0,1)$ on $\xi \in \mathbb{R}$. Plugging $R(\xi)$ into the $u$-th equation yields

$$
c R^{\prime}(\xi)-d R^{\prime \prime}(\xi)+\mu R(\xi)-\beta w(\xi) g(1-R(\xi))=0, \quad \forall \xi \in \mathbb{R} .
$$

It further follows from $R(-\infty)=0$ and the boundedness of $R$ that $\int_{-\infty}^{\xi} R(x) d x<+\infty$ on $\xi \leq 0$. Furthermore, integrating two sides of the above equality from $-\infty$ to $\xi \leq 0$ leads to

$$
c R(\xi)-d R^{\prime}(\xi)+\mu \int_{-\infty}^{\xi} R(x) d x-\int_{-\infty}^{\xi} \beta g(1-R(x)) w(x) d x=0 .
$$

Let $f(\xi)=\int_{-\infty}^{\xi} \beta w(x) g(1-R(x)) d x$ and $B_{0}(\xi)=\mu \int_{-\infty}^{\xi} R(x) d x$ for any $\xi \leq 0$. It is not difficult to show that $f(\xi) \leq C_{M} e^{\mu_{0} \xi}$ for any $\xi \in \mathbb{R}$, where $C_{M}>0$ is a constant. Based on the definition of $R(\xi)$, it has

$$
d R^{\prime}(\xi)-c R(\xi)=B_{0}(\xi)-f(\xi), \quad \xi \leq 0
$$

A directly computation causes to

$$
\begin{aligned}
R(\xi) & =\widehat{C}_{M} e^{\frac{c}{d} \xi}+\frac{1}{d} e^{\frac{c}{d} \xi} \int_{\xi}^{0} e^{-\frac{c}{d} \eta}\left(-B_{0}(\eta)+f(\eta)\right) d \eta \\
& =\widehat{C}_{M} e^{\frac{c}{d} \xi}+\frac{1}{d} e^{\frac{c}{d} \xi} \int_{\xi}^{0} e^{-\frac{c}{d} \eta} f(\eta) d \eta-\frac{1}{d} e^{\frac{c}{d} \xi} \int_{\xi}^{0} e^{-\frac{c}{d} \eta} B_{0}(\eta) d \eta \\
& \leq \widehat{C}_{M} e^{\frac{c}{d} \xi}+\frac{1}{d} e^{\frac{c}{d} \xi} \int_{\xi}^{0} e^{-\frac{c}{d} \eta} f(\eta) d \eta, \quad \forall \xi \leq 0
\end{aligned}
$$

where $\widehat{C}_{M}=R(0)$. In view of $f(\xi)=O\left(e^{\mu_{0} \xi}\right)$ as $\xi \rightarrow-\infty$, we get that $R(\xi)=O\left(e^{\mu_{0}^{\prime} \xi}\right)$ as $\xi \rightarrow-\infty$, where $\mu_{0}^{\prime}=\min \left\{\mu_{0}, c / d\right\}$. It follows from $0 \leq R(\xi)<1$ that

$$
\sup _{x \in \mathbb{R}}\left\{R(x) e^{-\mu_{0}^{\prime} x}\right\}<+\infty .
$$

On the basis of the above discussion, define the two-sided Laplace transform of $v$ and $w$ by

$$
\mathcal{L}_{0}(\lambda)=\int_{-\infty}^{+\infty} e^{-\lambda \xi} v(\xi) d \xi, \quad \mathcal{L}_{1}(\lambda)=\int_{-\infty}^{+\infty} e^{-\lambda \xi} w(\xi) d \xi
$$


We only take into account $\lambda \in \mathbb{R}_{+}$, too. $v(\xi)$ and $w(\xi)$ are bounded in $\mathbb{R}$, so it has

$$
\int_{0}^{+\infty} e^{-\lambda \xi} v(\xi) d \xi<+\infty \text { and } \int_{0}^{+\infty} e^{-\lambda \xi} w(\xi) d \xi<+\infty
$$

for any $\lambda \geq 0$. Therefor, we only consider

$$
\mathrm{L}_{0}(\lambda):=\int_{-\infty}^{0} e^{-\lambda \xi} v(\xi) d \xi, \quad \mathrm{L}_{1}(\lambda)=\int_{-\infty}^{0} e^{-\lambda \xi} w(\xi) d \xi
$$

Due to $v(\xi)>0$ and $w(\xi)>0$ for any $\xi \in \mathbb{R}$ and $\mathrm{L}_{i}(\cdot)$ is increasing in its domain, for each $i=0,1$, one of the following properties holds: (i) there is a positive constant $\nu_{i}>\mu_{0}$ so that $\mathrm{L}_{i}(\lambda)<+\infty$ for any $0 \leq \lambda<\nu_{i}$ and $\lim _{\lambda \rightarrow \nu_{i}-0} \mathrm{~L}_{i}(\lambda)=+\infty$; (ii) $\mathrm{L}_{i}(\lambda)<+\infty$ for any $\lambda \geq 0$.

In the following, we show $\nu_{i}=+\infty$, namely, for both $i=0,1, \mathcal{L}_{i}(\lambda)<+\infty$ for any $\lambda \geq 0$. First, we prove $\nu_{0}=\nu_{1}$ by a contradiction argument.

According to

$$
D_{v} v^{\prime \prime}(\xi)-c v^{\prime}(\xi)+(\beta g(1)+r) w(\xi)-\sigma v(\xi)=\beta(g(1)-g(u(\xi))) w(\xi), \quad \forall x \in \mathbb{R},
$$

it has

$$
\left(D_{v} \lambda^{2}-c \lambda-\sigma\right) \mathcal{L}_{0}(\lambda)+(\beta g(1)+r) \mathcal{L}_{1}(\lambda)=\beta \int_{-\infty}^{+\infty}(g(1)-g(u(\xi))) w(\xi) e^{-\lambda \xi} d \xi
$$

On the same way, we get

$$
\left(D \lambda^{2}-c \lambda-\delta\right) \mathcal{L}_{1}(\lambda)+\gamma \mathcal{L}_{0}(\lambda)=0
$$

which becomes to

$$
\left(-D \lambda^{2}+c \lambda+\delta\right) \mathcal{L}_{1}(\lambda)=\gamma \mathcal{L}_{0}(\lambda)
$$

If $\nu_{1} \neq \nu_{0}$, then it is a contradiction with (4.18). Thus, we can obtain $\nu_{0}=\nu_{1}:=\nu$. Secondly, we show $\nu=+\infty$ by using a contradiction way. Without loss of generality, let $\nu<+\infty$, namely, $\mathcal{L}_{i}(\lambda)<+\infty$ for any $0 \leq \lambda<\nu$ and $\lim _{\lambda \rightarrow \nu-0} \mathcal{L}_{i}(\lambda)=+\infty, i=0,1$. In fact, it is easy to see that $\left(-D \nu^{2}+c \nu+\delta\right)>0$ in 4.18$)$. Furthermore, if $\left(D_{v} \nu^{2}-c \nu-\sigma\right) \geq 0$ in (4.17), then by letting $\lambda \rightarrow \nu-0$, the left-hand side of (4.17) tends to infinity, however, based on (4.16) and (H1), the right-hand side of (4.17) is bounded, that is, it has

$$
\begin{aligned}
& \beta \int_{-\infty}^{+\infty}(g(1)-g(u(\xi))) w(\xi) e^{-\nu \xi} d \xi \\
\leq & \beta \mathcal{G} \sup _{\xi \in \mathbb{R}}\left\{(1-u(\xi)) e^{-\frac{\mu_{0}^{\prime}}{2} \xi}\right\} \int_{-\infty}^{+\infty} w(\xi) e^{-\left(\nu-\frac{\mu_{0}^{\prime}}{2}\right) \xi} d \xi \\
< & \infty
\end{aligned}
$$


where $\mathcal{G}=\sup _{x \in[0,1]}\left\{g^{\prime}(x)\right\}$ and $\mu_{0}^{\prime}$ has been defined in 4.16), which leads to a contradiction. If $\left(D_{v} \nu^{2}-c \nu-\sigma\right)<0$, then plugging 4.18) into 4.17) yields

$$
\left(\frac{m_{0}(\lambda, c) m_{1}(\lambda, c)}{\gamma}\left(\rho^{2}(\lambda, c)-1\right)\right) \mathcal{L}_{1}(\lambda)=\beta \int_{-\infty}^{+\infty}(g(1)-g(u(\xi))) w(\xi) e^{-\lambda \xi} d \xi
$$

for $0<\lambda<\nu$. It is obvious that $0<\nu<\lambda_{\max }(c)$. Similarly, when $\lambda \rightarrow \nu-0$, then the left-hand side of (4.19) tends to infinity, however, the right-hand side of 4.19 is bounded, which causes to a contradiction. Thus, the assumption $\nu<+\infty$ is also impossible.

Note that for $i=0,1, \mathcal{L}_{i}(\lambda)<+\infty$ for any $\lambda \geq 0$ has been proved. However, letting $\lambda \rightarrow+\infty$ in (4.17) yields a contradiction because $\lim _{\lambda \rightarrow+\infty}\left(D_{v} \lambda^{2}-c \lambda-\sigma\right)=+\infty$. The proof is completed.

\section{Numerical simulations}

In this section, we simulate traveling wave solutions for system 1.1) with $g(u)=u$ and $g(u)=u /(1+u)$, which connects the disease-free equilibrium $\left(u_{0}, v_{0}, w_{0}\right)$ and the endemic equilibrium $\left(u^{*}, v^{*}, w^{*}\right)$. For this purpose, the spatial domain $\mathbb{R}$ and the time domain $\mathbb{R}^{+}$ are truncated by $[0,800]$ and $[0,200]$, respectively and use piecewise functions as bellows as initial conditions:

$$
u(t, x)=\left\{\begin{array}{ll}
u^{*} & \text { if } 0 \leq x<400, t=0, \\
u^{0} & \text { if } 400 \leq x \leq 800, t=0,
\end{array} \quad v(t, x)= \begin{cases}v^{*} & \text { if } 0 \leq x<400, t=0, \\
0 & \text { if } 400 \leq x \leq 800, t=0\end{cases}\right.
$$

and

$$
w(t, x)= \begin{cases}w^{*} & \text { if } 0 \leq x<400, t=0 \\ 0 & \text { if } 400 \leq x \leq 800, t=0\end{cases}
$$

It further take Neumann boundary condition for system 1.1.

Case 1: $g(u)=u$. We take the following parameters of the model:

$$
\mu=0.65, \quad \beta=1.8, \quad \lambda=0.13, \quad \sigma=0.8, \quad r=0.6, \quad \delta=0.6, \quad \gamma=0.5
$$

It follows from these parameters that the basic reproduction number $R_{0} \approx 10>1$, the minimal speed $c^{*} \approx 0.244$, the disease-free equilibrium $(5,0,0)$ and the endemic equilibrium $(0.2029,2.0574,1.7085)$ are obtained.

Figure 5.1, which is the simulation results of system (1.1) with the given parameters, illustrates that system (1.1) admits a traveling wave solution $(u, v, w)$ with the minimal wave speed $c=c^{*}$. Furthermore, traveling wave of system (1.1) is not monotone, see Figures 5.2 and $5.5(\mathrm{a})$. 

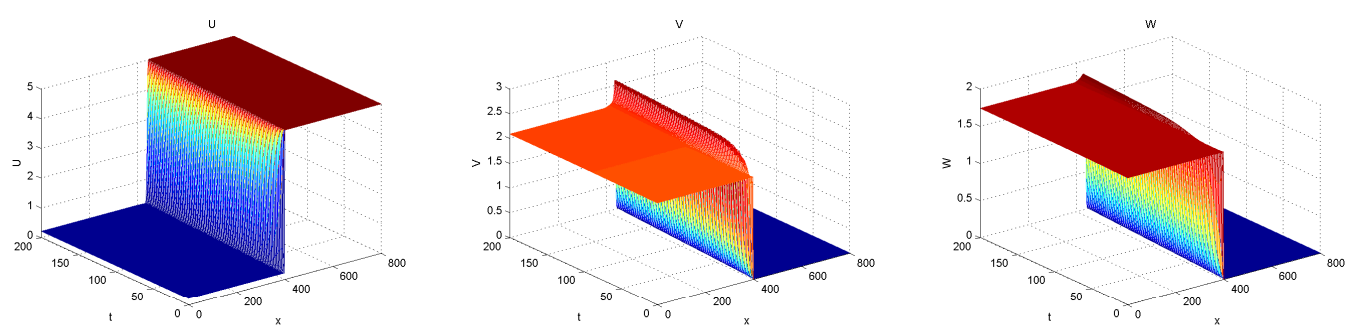

Figure 5.1: Numerical simulations of solutions for system (1.1) with $g(u)=u$.
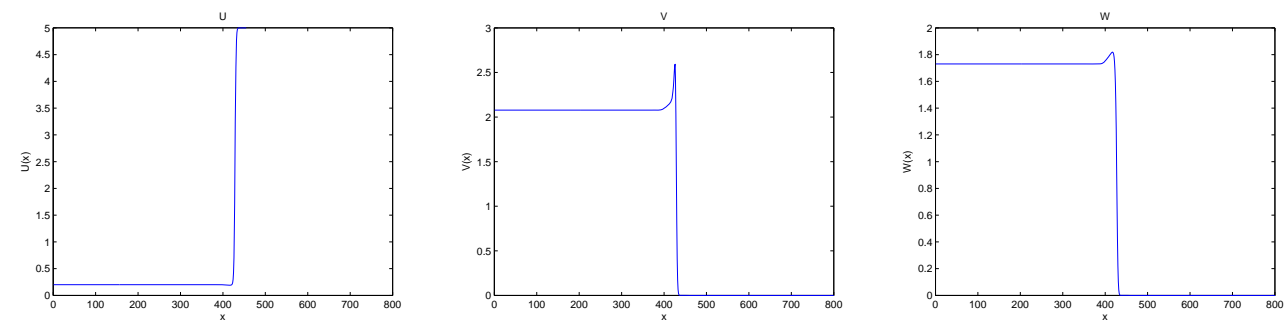

Figure 5.2: Cross section curves of solutions of system 2.1 at $t=150$ in Figure 5.1.

Case 2: $g(u)=u /(1+u)$. Here, we take the following parameters:

$$
\mu=1, \quad \beta=1.2, \quad \lambda=0.16, \quad \sigma=0.8, \quad r=0.5, \quad \delta=0.6, \quad \gamma=0.5 .
$$

By a straightforward computation, it has the basic reproduction number $R_{0} \approx 8>1$, the minimal speed $c^{*} \approx 0.12$, the disease-free equilibrium $(1.16,0,0)$ and the endemic equilibrium $(0.3753,2.495,2.0979)$.

Similarly, Figure 5.3 shows the existence of traveling wave solutions of system (1.1) with $c=c^{*}$ and Figures 5.4 and 5.5(b) demonstrate the non-monotonicity of the traveling wave solutions.
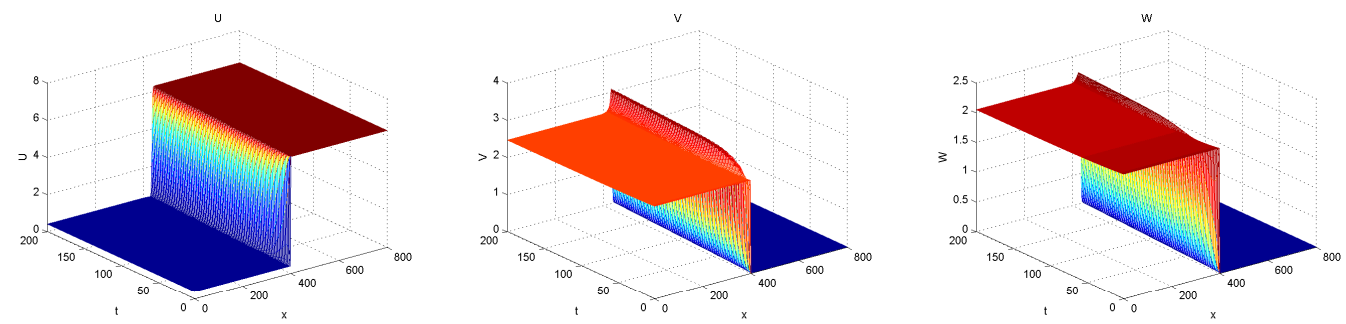

Figure 5.3: Numerical simulations of solutions for system 1.1$)$ with $g(u)=u /(1+u)$. 

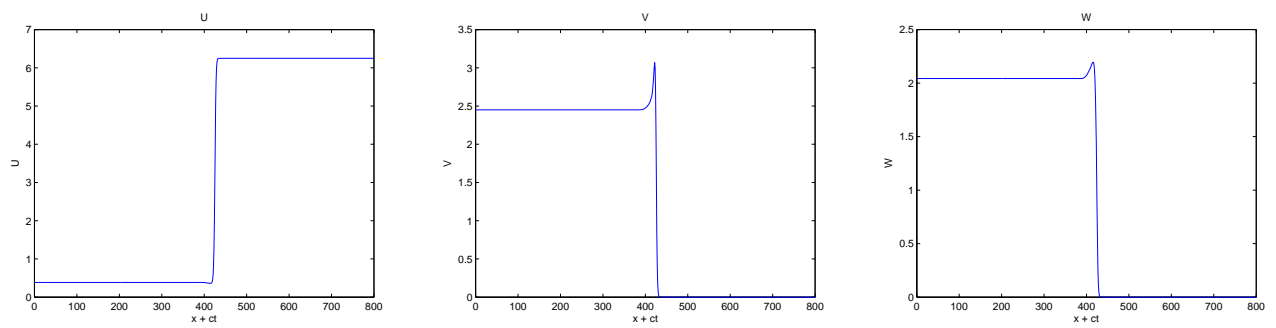

Figure 5.4: Cross section curves of solutions of system (1.1) at $t=150$ in Figure 5.3.
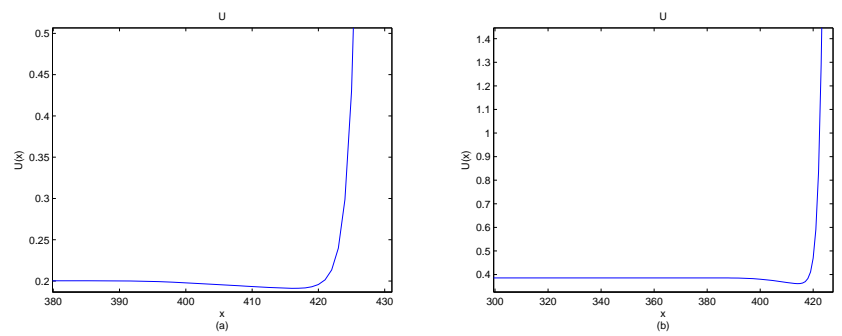

Figure 5.5: The value of $U(x)$ with $x \in(380,430)$ and $x \in(300,420)$ in Figures 5.2 and 5.4 respectively.

Another incidence rates can be treated similarly.

\subsection{Discussion}

In this paper, we considered a diffusive SEIR epidemic model with nonlinear incidence rate and constant recruitment and studied the existence and nonexistence of traveling wave solutions of the model. When the basic reproduction number $R_{0}>1$, we proved that there exists a number $c^{*}$ satisfying for each wave speed $c \geq c^{*}$, the system admits a nontrivial and nonnegative traveling wave solution and also investigated the nonexistence of nonnegative traveling wave solutions of the model when $R_{0}>1$ and $0<c<c^{*}$. Compared with [39], we consider the more general case, namely, we extend results for the nonexistence of traveling wave solutions of the system in 39 by using the Laplace transform.

\section{References}

[1] S. A. Al-Sheikh, Modeling and analysis of an SEIR epidemic model with a limited resource for treatment, Global Journal of Science Frontier Research Mathematics and Decision Sciences 12 (2012), 55-66. 
[2] R. M. Anderson, The Kermack-McKendrick epidemic threshold theorem, Bull. Math. Biol. 53 (1991), no. 1-2, 3-32.

[3] R. M. Anderson and R. M. May, Infectious Diseases of Humans: Dynamics and control, Oxford University Press, Academic, 1991.

[4] R. M. Anderson and W. Trewhella, Population dynamics of the badger (Meles meles) and the epidemiology of bovine tuberculosis (Mycobacterium bovis), Philos. Trans. Roy. Soc. London Ser. B 310 (1985), no. 1145, 327-381.

[5] F. Brauer, Compartmental models in epidemiology, in: Mathematical Epidemiology, 19-79, Lecture Notes in Mathematics 1945, Math. Biosci. Subser., Springer, Berlin, 2008.

[6] O. Diekmann, Run for your life: A note on the asymptotic speed of propagation of an epidemic, J. Differential Equations 33 (1979), no. 1, 58-73.

[7] B. Dubey, A. Patra, P. K. Srivastava and U. S. Dubey, Modeling and analysis of an SEIR model with different types of nonlinear treatment rates, J. Biol. Systems 21 (2013), no. 3, 1350023, 25 pp.

[8] A. Ducrot and P. Magal, Travelling wave solutions for an infection-age structured epidemic model with external supplies, Nonlinearity 24 (2011), no. 10, 2891-2911.

[9] A. Ducrot, P. Magal and S. Ruan, Travelling wave solutions in multigroup agestructured epidemic models, Arch. Ration. Mech. Anal. 195 (2010), no. 1, 311-331.

[10] S.-C. Fu and J.-C. Tsai, Wave propagation in predator-prey systems, Nonlinearity 28 (2015), no. 12, 4389-4423.

[11] D. Gilbarg and N. S. Trudinger, Elliptic Partial Differential Equations of Second Order, Classics in Mathematics, Springer-Verlag, Berlin, 2001.

[12] H. W. Hethcote, The mathematics of infectious diseases, SIAM Rev. 42 (2000), no. 4, 599-653.

[13] Y. Hosono and B. Ilyas, Traveling waves for a simple diffusive epidemic model, Math. Models Methods Appl. Sci. 5 (1995), no. 7, 935-966.

[14] W. Huang, Traveling waves for a biological reaction-diffusion model, J. Dynam. Differential Equations 16 (2004), no. 3, 745-765.

[15] G. Huang, Y. Takeuchi, W. Ma and D. Wei, Global stability for delay SIR and SEIR epidemic models with nonlinear incidence rate, Bull. Math. Biol. 72 (2010), no. 5, $1192-1207$. 
[16] A. Korobeinikov, Lyapunov functions and global stability for SIR and SIRS epidemiological models with non-linear transmission, Bull. Math. Biol. 68 (2006), no. 3, 615626.

[17]_ Global properties of infectious disease models with nonlinear incidence, Bull. Math. Biol. 69 (2007), no. 6, 1871-1886.

[18] _ Global properties of SIR and SEIR epidemic models with multiple parallel infectious stages, Bull. Math. Biol. 71 (2009), no. 1, 75-83.

[19] A. Korobeinikov and P. K. Maini, Non-linear incidence and stability of infectious disease models, Math. Med. Biol. 22 (2005), no. 2, 113-128.

[20] Y. Li, W.-T. Li and F.-Y. Yang, Traveling waves for a nonlocal dispersal SIR model with delay and external supplies, Appl. Math. Comput. 247 (2014), 723-740.

[21] W. M. Liu, S. A. Levin and Y. Iwasa, Influence of nonlinear incidence rates upon the behavior of SIRS epidemiological models, J. Math. Biol. 23 (1986), no. 2, 187-204.

[22] M. Martcheva, An Introduction to Mathematical Epidemiology, Texts in Applied Mathematics 61, Springer, New York, 2015.

[23] J. D. Murray, Mathematical Biology, Biomathematics 19, Springer-Verlag, Berlin, 1989.

[24] S. Ruan, Spatial-temporal dynamics in nonlocal epidemiological models, in: Mathematics for Life Science and Medicine, 97-122, Biol. Med. Phys. Biomed. Eng., Springer, Berlin, 2007.

[25] S. Ruan and W. Wang, Dynamical behavior of an epidemic model with a nonlinear incidence rate, J. Differential Equations 188 (2003), no. 1, 135-163.

[26] C. Sun, Y. Lin and S. Tang, Global stability for an special SEIR epidemic model with nonlinear incidence rates, Chaos Solitons Fractals 33 (2007), no. 1, 290-297.

[27] B. Tian and R. Yuan, Traveling waves for a diffusive SEIR epidemic model with standard incidences, Sci. China Math. 60 (2017), no. 5, 813-832.

[28] _ Traveling waves for a diffusive SEIR epidemic model with non-local reaction and with standard incidences, Nonliear Anal. Real World Appl. 37 (2017), 162-181.

[29] _ Traveling waves for a diffusive SEIR epidemic model with non-local reaction, Appl. Math. Model. 50 (2017), 432-449. 
[30] P. van den Driessche and J. Watmough, Reproduction numbers and sub-threshold endemic equilibria for compartmental models of disease transmission, Math. Biosci. 180 (2002), 29-48.

[31] Z.-C. Wang and J. Wu, Travelling waves of a diffusive Kermack-McKendrick epidemic model with non-local delayed transmission, Proc. R. Soc. Lond. Ser. A Math. Phys. Eng. Sci. 466 (2010), no. 2113, 237-261.

[32] Z.-C. Wang, J. Wu and R. Liu, Traveling waves of the spread of avian influenza, Proc. Amer. Math. Soc. 140 (2012), no. 11, 3931-3946.

[33] Z. Wang and $\mathrm{R}$. Xu, Travelling waves of a diffusive epidemic model with latency and relapse, Discrete Dyn. Nat. Soc. 2013 (2013), Art. ID 869603, 13 pp.

[34] J. Wu and S. Ruan, Modeling spatial spread of communicable diseases involving animal hosts, in: Spatial Ecology, 293-316, Chapman \& Hall/CRC, Boca Raton, FL, 2009.

[35] H. Xiang, Y. Wang and H. Huo, Analysis of the binge drinking models with demographics and nonlinear infectivity on networks, J. Appl. Anal. Comput. 8 (2018), no. 5, 1535-1554.

[36] Z. Xu, Traveling waves for a diffusive SEIR epidemic model, Commun. Pure Appl. Anal. 15 (2016), no. 3, 871-892.

[37] _ Traveling waves in an SEIR epidemic model with the variable total population, Discrete Contin. Dyn. Syst. Ser. B 21 (2016), no. 10, 3723-3742.

[38] Y. Yang and D. Xiao, Influence of latent period and nonlinear incidence rate on the dynamics of SIRS epidemiological models, Discrete Contin. Dyn. Syst. Ser. B 13 (2010), no. 1, 195-211.

[39] L. Zhao and Z.-C. Wang, Traveling wave fronts in a diffusive epidemic model with multiple parallel infectious stages, IMA J. Appl. Math. 81 (2016), no. 5, 795-823.

[40] L. Zhao, Z.-C. Wang and S. Ruan, Traveling wave solutions in a two-group epidemic model with latent period, Nonlinearity 30 (2017), no. 4, 1287-1325.

Lin Zhao

Department of Applied Mathematics, Lanzhou University of Technology, Lanzhou, Gansu 730050, P. R. China

E-mail address: zhaolin@lut.cn 
Liang Zhang

School of Mathematics and Statistics, Lanzhou University, Lanzhou, Gansu 730000, P. R. China

E-mail address: lz@lzu.edu.cn

Haifeng Huo

Department of Applied Mathematics, Lanzhou University of Technology, Lanzhou, Gansu 730050, P. R. China

E-mail address: huohf70@sohu.com 